\title{
Knowing Hands Converse with an Expressive Body - An experience of osteopathic touch
}

Seth Consedine

A thesis submitted in partial fulfilment of the requirements for the degree of Master of Osteopathy, Unitec New Zealand, 2007 


\section{(U) Unitec}

\section{Declaration}

\section{Name of candidate: Seth Consedine}

This Thesis/Dissertation/Research Project entitled Knowing Hands Converse with an Expressive Body: An experience of osteopathic touch is submitted in partial fulfilment for the requirements for the Unitec degree of Master of Osteopathy

\section{CANDIDATE'S DECLARATION}

I confirm that:

- This Thesis/Dissertation/Research Project represents my own work;

- The contribution of supervisors and others to this work was consistent with the Unitec Regulations and Policies.

- Research for this work has been conducted in accordance with the Unitec Research Ethics Committee Policy and Procedures, and has fulfilled any requirements set for this project by the Unitec Research Ethics Committee.

Research Ethics Committee Approval Number: 2006.600

Candidate Signature:

Date:

Student number: 1131525 


\section{Abstract}

Aim - The aim of this phenomenological study was to examine and describe the patient's experience of touch during a consultation with an osteopathic practitioner.

Background - Touch is the process by which one person physically contacts another individual and as such plays an integral role in osteopathic practice. Osteopathic practitioners use touch in almost every facet of care including examination, diagnosis and treatment. Although this form of communication is intrinsic to osteopathic practice, there has been no research undertaken to date that qualifies the experience of osteopathic touch within a treatment session.

Methods - Three local osteopaths identified five potential participants from their own practice. Data were recorded during five face-to-face semi-structured interviews that were conducted by the primary researcher. Interviews were transcribed and the textual data was analysed using the principles of van Manen's (1997) hermeneutic phenomenology. Using this approach, significant themes were identified in the data and a description of the lived experience of osteopathic touch emerged.

Results - Three phenomenological themes were identified in the data and broken down into nine constituent subthemes. Theme [A] The Process - a physical interaction, drew attention to the physical patient-practitioner relationship and the physical and psychological implications of this interaction. Theme [B] Professionalism $-a$ practitioner's responsibility explored the role of touch in developing professional boundaries and emphasised the patient's expectations surrounding the physical contact, while Theme [C] Reassurance - a therapeutic necessity examined the role of touch in reassuring the patient about their presenting complaint and the practitioner's medical knowledge and technical skill.

Conclusions - The experience of touch for the participants in an osteopathic session is one of care and security. For the participants touch plays a critical role in the therapeutic relationship by supporting and validating their experiences and by communicating practitioner humanism and professionalism. Furthermore, the experience of touch was identified as a critical component of the therapeutic interaction. Indications for further research and implications for the osteopathic profession are discussed. 


\section{Acknowledgements}

First and foremost I would like to acknowledge and thank the participants of this study. I am grateful that you were able to talk to me, a complete stranger, about your osteopathic treatment and consider it an honour that you shared your experiences with me. Needless to say that without people like you, research like this would not be possible and the literature and profession would be poorer for it.

Secondly I would like to individually acknowledge my supervisors, each of whom has been invaluable, for their contribution to this study. To Dr Elizabeth Niven, your tireless work in stimulating my interest, challenging my ideas, proof reading, motivating and supporting me throughout the research process has been stimulating and enjoyable. To Associate Professor Clive Standen, your practical ideas, osteopathic perspective and proof reading skills have been insightful and helpful. And lastly, to Dr Craig Hilton, your open door, thoughtful remarks, challenging persona, and proof reading skills have kept me honest throughout the research process and have been an important contribution.

Thirdly I would like to acknowledge and thank my family. To my parents, you have nurtured and supported my ongoing interest in people and the complex phenomenon of the therapeutic interaction. To my twin Anna, your proof reading skills and support have been offered with an openness and generosity which characterises who you are. To my older brother Nathan, your tutorials on writing, research methods and processes have equipped me with skills which underpin this research project.

Fourthly, I would like to thank two members of Unitec general staff; Cynthia Almeida of the Post Graduate Centre for her administrative skills and dedication and loyalty to the interests of the Post-Graduate students, and Cathy O'Brien for her tireless efforts to master Endnote.

Finally, I would like to acknowledge and thank my partner and best friend Kate. Not only have you contributed to this research project with ideas and proof reading but your presence and love have given me strength more times than I can remember. 


\section{Table of Contents}

Abstract __ iii

Acknowledgements iv

Table of Contents__v

Chapter One: The Study Topic____ 1

Introduction __ 2

Definitional and Conceptual Issues __ 3

The Area of Research __ 4

My Personal Interest in the Topic ___ 5

Rationale for the Research _ 6

Chapter Two: Literature Review _ 8

Touch and Development __ 9

Touch and Attachment __ 11

Touch and Communication _ـ 12

Patient-Practitioner Communication _ـ 14

The Philosophy of the Body and Touch ___ 15

Touch and Psychology _ـ 17

Touch, Culture and Healthcare __ 18

Touch and Nursing _ 20

Touch and the Manual Therapies __ 21

Touch and Osteopathy __ 24

Chapter Three: Methodology___ 27

The Importance of Research Methodology __ 28

Why Qualitative? _ 29

Why Phenomenology?___ 31

Husserlian vs Heideggerian Phenomenology __ 32

Hermeneutic Phenomenology__ 33

The Question of Rigour in Qualitative Research __ 34

Chapter Four: Undertaking the Study ___ 36

Approval Process, Ethical Considerations and Sampling _ 37

Data Gathering: The Interview ___ 39

Transcription and Data Analysis___ 40

Maintaining Rigour __ 44

Introduction to the Study's Participants ___ 46 
A Guide to Reading Chapter Five 46

Chapter Five: Data Analysis 48

THEME [A]: The Process - a physical interaction 49

[A1] Engagement - let the session begin 50

[A2] Dialogue - knowing hands converse with an expressive body __ 52

[A3] Support - just holding 54

THEME [B]: Professionalism - a practitioner's responsibility 57

[B1] Care - an empathic quality 58

[B2] Trust - a prerequisite for physical contact 61

[B3] Boundaries - a professional relationship 63

THEME $[C]$ - Reassurance: a therapeutic necessity 67

[C1] Knowledge - with seeing fingers

[C2] Competence - a knowing touch 70

[C3] Confidence - very reassuring 72

Summary 73

Chapter Six: Discussion 74

THEME [A]: The Process - a physical Interaction

[A1] Engagement - let the session begin

[A2] Dialogue - knowing hands converse with an expressive body 76

[A3] Support - just holding 79

THEME [B]: Professionalism - a practitioner's responsibility 85

[B1] Care - an empathic quality 85

[B2] Trust - a prerequisite for physical contact 88

[B3] Boundaries - a professional relationship 91

THEME [C]: Reassurance - a therapeutic necessity ___ 93

$[\mathrm{C1}$ K Knowledge - with seeing fingers ___ 94

[C2] Competence - a knowing touch 95

[C3] Confidence - very reassuring _ 98

Summary _ 101

Chapter Seven: Concluding Thoughts__ 102

Strengths of the Study _ 103

Limitations of the Study _ 104

Directions for Future Research _ 106

Implications for Education and Practice___ 107

A Final Word _ 109

References 110

Appendices_ 124

Appendix A - Ethical Approval___ 125

Appendix B - Inclusion/Exclusion Criteria __ 126

Appendix C - Information Sheet for Participants

Appendix D - Consent Form _ 128

Appendix E - Information about Researcher _ 129

Appendix F - Interview Schedule __ 130 


\section{Chapter One: The Study Topic}


This chapter begins by introducing the topic and exploring some definitional and conceptual issues surrounding touch in the literature. This is followed by a brief outline of the research area and an explanation of my own interest in touch. The second chapter reviews and examines some of the diverse literature that has investigated the phenomena of touch focusing on broad areas of human existence and in particular on the literature within the physical therapies. The third and fourth chapters outline the research methodology and philosophy that guided this study and the process that was undertaken to complete the project. The fifth and sixth chapters respectively analyse and discuss the themes that emerged from the data. The seventh chapter concludes with the study's limitations, directions for further research, the main findings of the study and the implications for osteopathic practice.

\section{Introduction}

Patients of osteopathy are examined, diagnosed and treated all with the aid of physical contact (or touch) between themselves and their osteopath. Thus this form of touch represents an intrinsic part of osteopathic practice. Surprisingly however, the examination of touch is underrepresented in the osteopathic literature.

The current study set out to explore the phenomena of touch (see definition section below) within the osteopathic session. Data collection involved interviewing five patients of osteopathy about their experiences of touch within an osteopathic session. The data from the interviews was subsequently analysed utilising hermeneutic philosophy and methodology. Analysis of the data suggested that touch plays an integral role in mediating the patient-practitioner communication and hence the therapeutic process. 


\section{Definitional and Conceptual Issues}

As this thesis focuses on the phenomena of touch it is necessary to define and conceptualize this term. The Concise Oxford English Dictionary $\left(11^{\text {th }}\right.$ ed, revised) defines touch as to "come into or be in contact with; come or bring into mutual contact" and "bring one's body into contact with" (Soanes \& Stevenson, 2006, p. 1523). While this simple description focuses on the physical procedure of touching an object the Dorland's Illustrated Medical Dictionary $\left(30^{\text {th }}\right.$ Ed.) describes touch within a biomechanical framework "the sense (actually group of senses) by which contact with objects gives evidence as to certain of their qualities, as registered by mechanoreceptors in the skin and mucous membranes" (Dorland, 2003, p. 1925). Hertenstein (2002) acknowledges the semantically rich nature of touch by dividing touch into two highly dissociable phenomena: "(a) the action of an object on the skin and (b) the registration of information by the sensory systems of the skin (what in common usage we call feeling)" (p. 71).

Interestingly, none of these definitions specifically identifies touch as a physical process between two people and thus none of these descriptions taken alone adequately expresses the meaning of touch as it will be used within this thesis. For the purposes of the current study touch will include all physical contact that occurs between the osteopathic practitioner and the patient. This will include both necessary touch, defined as "deliberate physical contact for the performance of a certain task" (Routasalo, 1999, p.844) and non-necessary touch, defined as "relatively spontaneous and affective [touch], such as patting a patient on the arm" (Routasalo, 1999, p.844). ${ }^{1}$ The current definition also acknowledges the reciprocal nature of touch whereby the act of touching is to be touched.

\footnotetext{
${ }^{1}$ However, this does not necessarily exclude touch that may be unwanted or have negative connotations.
} 


\section{The Area of Research}

There is a general embryological law that states that the earlier a function develops the more fundamental that function is likely to be (Montagu, 1986). In the human embryo, the most primitive and basic sense to develop is the tactile sense (Barnard \& Brazelton, 1990; Frank, 1957; Montagu, 1986; Nathan, 1999). Furthermore the primary organ of tactility and indeed the organ which separates and differentiates an individual from the rest of their environment is the skin. The skin not only provides humans with a basic means for interacting with and responding to the world, it is also intimately related to an individual's identity and sense of self (Frank, 1957; Montagu, 1986). This idea is most evident in early life where touch plays an essential role in an infant's growth, development and health but is also apparent later in life when touch can influence and mediate an adult's physical and mental well-being (Bowlby, 1988; Field, 1998; Lederman, 2005).

While the intuitive benefits of touch to both healthy and ailing individuals have perhaps been known for several millennia, touch has become the subject of experimental research only in the last sixty years (Field, 2001; Montagu, 1986; Ventegodt, Morad, \& Merrick, 2004). Beginning in the first half of the twentieth century, researchers began to look at the effect of physical contact on different domains of human existence including the physical, mental and spiritual realms of life. However, a read through some of this early research reveals a consistent argument that there is a dearth of scientific research into the meaning, use and potential benefits of touch and that further research is warranted (Frank, 1957; Harlow, 1958). Interestingly, this theme continues into more contemporary papers, with authors from fields including psychology, nursing and manual medicine still calling for more scientifically conducted research into the benefits of touch and its potentially therapeutic uses (Estabrooks, 1989; Field, 2000; Helm, Kinfu, Kline, \& Zappile, 1997; Hertenstein, 2002; Nathan, 1993). 


\section{My Personal Interest in the Topic}

I am a thirty-year-old pakeha of Irish heritage born in Christchurch into a middle class working family. I was raised within a caring environment that fostered a sense of connection to the family, the community and later on to humanity and the greater environment.

I enjoy the act of touching. I believe touch is an important way of connecting with people and is capable of contributing to a sense of belonging (to groups, families, friends). I believe that touch helps define a person and enables them to identify themselves in the world. I also believe the act of touching is capable of eliciting powerful emotions in both the toucher and those being touched.

I think my initial interest in this area of research can be traced back to the many fruitful conversations I have had with my parents about therapeutic relationships. As both my parents worked in the field of human relations I think I have naturally become curious about the dynamics of the therapeutic relationship created between the healthcare professional and their patient. While a verbal dialogue mediates the therapist-patient relationship in psychotherapy, for physical therapists the interaction is mediated by the way in which they touch their patients. What began to interest me is to what extent does the physical contact between the practitioner and the patient influence and affect the therapeutic process?

My interest in this area of osteopathy was further piqued by some cranial treatments I received early in my undergraduate degree. These treatments consisted of me lying on my back and the practitioner cradling my head for approximately half an hour. What struck me about these sessions was that while I was unable to feel the subtle cranial techniques that she utilised, I came away from her clinic feeling integrated, held and profoundly cared for. While it may be argued that this was due to the cranial technique itself this experience planted in me a seed of interest about the beneficial or therapeutic outcomes which can be attributed to the physical contact which is created between the practitioner and the patient during an osteopathic session. 
My undergraduate training also exposed me to research that questioned the efficacy of osteopathic treatment. Given the substantial amount of research that exists questioning the value or benefit of this healthcare modality, why do individuals continue to seek the advice of osteopathic practitioners? My supposition is that it is not merely that the individuals are unaware of this research but that measurable therapeutic outcomes are not the patient's sole reason for consulting healthcare practitioners. Furthermore, I believe that a therapeutic relationship that is mediated by physical contact could offer part of the answer to why patients continue to consult osteopathic practitioners and other physical therapists.

\section{Rationale for the Research}

Touch is an intrinsic part of manual therapy ${ }^{2}$ and for most practitioners plays a large role in communicating, diagnosing, and treating a patient. Furthermore, within the current medical model there is a requirement by both the public and funding bodies to use the best evidence based practices. However this prerequisite not only requires professions to provide evidence of the most effective diagnostic and treatment techniques but also requires them to investigate and broadly understand how their mode of healthcare is effective.

As with other areas of healthcare, osteopathic touch has been largely neglected as a subject for research. While some osteopathic authors have highlighted the huge significance of touch in osteopathy, notably Bevis Nathan (1999) and Eyal Lederman (2005), their books draw heavily on research from other disciplines and provide only anecdotal evidence from their own practices about the importance of touch to this field of medicine.

\footnotetext{
${ }^{2}$ In the current study manual therapy will refer to all therapies which use manipulative techniques including physiotherapy, osteopathy and chiropractic
} 
This chapter has briefly introduced the topic of touch and outlined how the current research paper examines the role of touch in an osteopathic session. Following this the chapter examined some definitional and conceptual issues surrounding touch and briefly introduced the idea of touch in the literature. To give the reader some perspective on the research, the author's interest in this area of research was also elucidated and to conclude the chapter a brief rationale for this research project was given. Chapter two will now review the broad literature base examining touch and highlight the scarcity of research examining this important area of osteopathic practice. 


\section{Chapter Two: Literature Review}


Touch affects almost every sphere of human existence. Given the universal nature of touch and the tactile sense this literature review sets out to examine the impact touch has had on human existence. In an attempt to organise this broad research base into a coherent structure the review will begin by examining the influence of touch in the psychological literature starting with its impact on early development and bonding and progressing to its use in communication and psychotherapy later in life. The review then briefly examines how touch is viewed in the philosophical literature before considering its cultural underpinnings. To conclude the review touch is explored in the healthcare literature, beginning with an overview of the extensive nursing literature and finishing with a more specific examination of the physical therapies literature.

\section{Touch and Development}

As noted above, the tactile system is one of the earliest and most primitive senses the human embryo develops (Montagu, 1986). In utero, the foetus is surrounded and supported by amniotic fluid and consequently early tactile stimulation involves constantly fluctuating pressure waves which correspond to the mother's many physiological processes (Field, 2001; Montagu, 1986; Nathan, 1999). However, this harmonious internal environment is abruptly disrupted as the baby is powerfully propelled along the birth canal during parturition (Montagu, 1986). The biophysicist Frederick Sachs (1988) describes a child's initiation into the world as a "kaleidoscope of tactile sensations: the constrictions of the birth canal along the length of her body; the firm grip of the physician's hands on her body, shoulders and ankles; the tender strokes of her mother's fingertips on her forehead and cheeks" (p.28). ${ }^{3}$ It is through this process however that the infant is introduced to an expectant world that is rich in more novel and challenging tactile experiences.

\footnotetext{
${ }^{3}$ Montagu (1986) has also noted that cesarean-delivered babies suffer from a number of disadvantages from the moment of birth including a higher mortality rate, respiratory disorders, greater lethargy and altered biochemical responses (serum glucose regulation) as well as significantly greater emotional disturbances than their vaginally delivered counterparts.
} 
The importance of touch and early development has been classically illustrated with Harlow's experiments with infant monkeys. These studies involved careful observation of infant macaque monkeys and their behaviour in response to an artificial inanimate mother (Harlow, 1958). What Harlow discovered was that their need for "contact comfort" ${ }^{\prime 4}$ outweighed their need for either food or warmth. In fact the results were so remarkable that he concluded that "the primary function of nursing as an affectional variable is that of ensuring frequent and intimate body contact of the infant with the mother" (Harlow, 1958, p.77). Furthermore, Harlow argued that the monkey's need for contact comfort was intimately connected with attachment (see below) and the provision of maternal love.

The findings of Harlow's early observations have since permeated through both psychological and medical literature. Of particular interest in the medical field is the employment of kangaroo care $(\mathrm{KC})$ in neonatal intensive care units with preterm and low birth weight infants. $\mathrm{KC}$ is defined as skin-to-skin contact between a mother and her newborn and may begin soon after birth or be delayed for several weeks (CondeAgudelo, Diaz-Rossello, \& Belizan, 2003; DiMenna, 2006). Proponents of KC believe that it promotes weight gain, decreases hospital stay, increases skin temperature, maintains higher oxygen saturation, increases physiological stability including heart and respiratory rate, increases sleep time and decreases irritability of preterm and low birth weight neonates (McCain, Ludington-Hoe, Swinth, \& Hadeed, 2005; Rojas et al., 2003). However, a recent Cochrane Review of $\mathrm{KC}$ for low birth weight infants concluded that while KC improved mother-infant bonding and reduced severe illness, infection, breast-feeding problems and maternal dissatisfaction with method of care there was still not enough evidence to suggest that $\mathrm{KC}$ is an effective alternative to standard care (Conde-Agudelo et al., 2003).

Along with a growing interest in the use of Kangaroo Care there has been a concurrent increase in developmental literature about the benefits of neonatal massage therapy (Blackwell, 2000). According to Dr Tiffany Field (1998) of the Miami Touch Institute, regular neonatal massage can have both important psychological and physiological results in full term and preterm infants. In support of this assertion Field cites a number

\footnotetext{
${ }^{4}$ Physical contact for reassurance
} 
of studies conducted at the Institute in which both preterm or full-term infants have significantly increased weight, decreased cortisol levels and increased motor activity following massage or touch therapy.

While Field's (1998) conclusions appear intuitive, given Harlow's experimental findings, controversy still exists about the use of massage for preterm infants. For example a recent Cochrane review concluded that while preterm infant massage may improve weight gain and decrease the length of hospital stay, the evidence is weakened due to methodological faults in the reviewed trials (Vickers, Ohlsson, Lacy, \& Horsley, 2004). Their conclusion was that the overall evidence for improved developmental outcomes following preterm infant massage was weak and did not warrant wider use. When considering the Cochrane review however it should be remembered that their primary question was "whether preterm and/or LBW (low birth weight) infants exposed to massage experience improved weight gain and earlier discharge compared to those infants receiving standard neonatal care" (Vickers et al., 2004, p. 2). Consequently the paper only reviewed studies that examined this particular question. While this improves the specificity of the review it has potentially limited its clinical utility by ignoring other potential benefits of the massage therapy such as improved developmental outcomes or parent satisfaction.

\section{Touch and Attachment}

Harlow's innovative ideas during the 1950s were also picked up and developed in the psychological literature by a contemporary researcher John Bowlby. Bowlby's attachment theory has been described by some as one of the most important theoretical developments in psychoanalysis since Freud (Holmes, 1993). Consistent with Harlow's observations, a key feature of Bowlby's attachment theory is that the quality of parentinfant physical contact is central in the development of a secure, care-giving environment (Hertenstein, 2002). According to Bowlby (1988) attachment behaviour "is any behaviour that results in a person attaining or maintaining proximity to some other clearly defined individual who is conceived as better able to cope with the world" 
(p.26). Bowlby also believed that attachment behaviour is in no way restricted to the very young, but is observable in adolescents and adults of both sexes, particularly under times of stress or anxiety. Moreover, given the limited availability of one's parent in adulthood an individual may seek a surrogate parent for comfort when distressed later in life.

Central to Bowlby's (1969) attachment theory was the idea that attachment behaviour is primarily developed in accordance with the experiences a person had in their family of origin. Furthermore it is through these early tactile experiences that an individual learns an association between physical contact and the feeling of physical and psychological well-being. Nathan (1999) argues that in our adult lives emotio-kinaesthetic memories are recorded in our body tissues which remember every detail of our mother's holding, caressing and rocking and the associated feelings of self worth. This idea also has an embryological origin, as the skin and the nervous system arise from the same embryonic tissue, the ectoderm (Montagu, 1986). Consistent with these concepts, some contemporary authors believe that early touch experiences inevitably go a long way in shaping some of the profound psychological responses which physical therapy frequently provokes later in life and that health practitioners must consider this every time they engage with a patient (Lederman, 2005; Milne, 1995; Nathan, 1999).

\section{Touch and Communication}

While touch is a rich and expressive form of communication, the communicative functions of touch between humans have historically been largely overlooked in the research literature (Hertenstein, 2002). In explanation Hertenstein offers both philosophical and methodological reasons for this scarcity. In the past, philosophers believed that the sense of vision offered the most clear and uncensored perception of the world. As a consequence both philosophers ${ }^{5}$ and scientists have neglected other senses and focused their thought and energy into the investigation of vision. Hertenstein also

\footnotetext{
${ }^{5}$ Hertenstein has acknowledged that the writing of the philosopher Merleau-Ponty (1962) is an exception to this trend.
} 
states that the primary methodological reason for this paucity of research is that touch is an incredibly complex sense and is thus a difficult modality to study. In a later review Hertenstein, Verkamp, Kerestes and Holmes (2006) also added that much touching occurs in private and is thus difficult for researchers to observe and that the taboos against touching among humans make it difficult to study in a laboratory setting.

While historically the communicative functions of touch have been overlooked, Hertenstein et al. (2006) have conceded that the communicative functions of touch have gained increasing attention in the literature over the last three decades. To support this they have written an extensive article reviewing the literature on the communicative functions of touch in humans, nonhuman primates and rats. While much of the review is beyond the scope of this discussion there are a number of findings that are relevant to the current study.

Firstly Hertenstein et al. (2006) have reviewed the communicative functions of touch in infancy, particularly with regard to its role in emotional communication, attachment and bonding. Of particular interest, the authors reported a number of studies that indicate that touch communicates emotion, particularly the hedonics ${ }^{6}$ of emotion. For example in infancy the authors reported that touch communicates positive emotions and adds to the positive reinforcement value of other forms of communication (e.g. verbal communication). In addition, studies have indicated that touch is both capable of communicating and modulating negative emotions between individuals.

Secondly Hertenstein et al. (2006) reviewed the communicative functions of touch among human adults. ${ }^{7}$ More specifically the review focused on three major domains in which touch plays an important communicative role in adult life: compliance, power relations and affective phenomena. Of particular interest to this research was the review of the role of touch in the communication of power and affective phenomena. With regard to the communication of power the authors found that the research suggests that people who initiate touch are perceived by others as more powerful and of higher status

\footnotetext{
${ }^{6}$ Pleasant or unpleasant sensation

${ }^{7}$ While Hertenstein and colleagues (2006) have focused on the communicative functions of touch they have not focused on ritualistic (handshake) or instrumental (touching them in an attempt to show someone how to do something) touch.
} 
than those who do not. As with infancy, touch is also important in the communication of emotions in adulthood. In particular Hertenstein et al. (2006) review literature that suggests touch has the capacity to communicate intimacy, hedonic emotions and liking. Moreover, the perceived quality of different types of touch is mediated by gender, age, relationship (between toucher and those touched), status, intentionality and the context (the setting in which tactile interactions occur).

\section{Patient-Practitioner Communication}

The patient-practitioner interaction is one of the most complex and challenging interpersonal relationships an individual has to navigate and healthcare professionals are therefore required to develop and maintain a high standard of interpersonal communication (Ayonrinde, 2003; Ong, De Haes, Hoos, \& Lammes, 1995; D. Williams, 1997). This is due to the large number of activities in the patient-practitioner interaction which demand a high level of communication skills including interviewing, explaining, giving instructions and advising. More importantly, the general practice, nursing and physical therapies literature has identified patient-practitioner communication as an important but often poorly performed feature of the patientpractitioner relationship (Barrier, Li, \& Jensen, 2003; Bendapudi, Berry, Frey, Parish, \& Rayburn, 2006; Fagerberg, Kragstrup, Støvring, \& Rasmussen, 1999; Gyllensten, Gard, Salford, \& Ekdahl, 1999; Kelly, 2007; Potter, Gordon, \& Hamer, 2003; Reifsteck, 1998).

In the literature communication is classically divided into verbal and non-verbal behaviours (Ong et al., 1995; D. Williams, 1997). Research has suggested that nonverbal behaviours comprise between $55 \%$ and $95 \%$ of the communication in any given interaction (Roberts \& Bucksey, 2007). Williams (1997) has identified seven forms of non-verbal communication utilised within healthcare settings - proximity, touch, eye contact and eye-gaze, facial expression, gesture, body posture and head movements. She has argued that these behaviours serve several functions including giving information, seeking information, expressing emotions, communicating interpersonal 
attitudes, establishing and maintaining relationships and regulating social interactions. In addition, it is evident that illness often creates fear, anxiety and emotional uncertainty in patients and thus a heightened awareness to subtle non-verbal clues that the practitioner may communicate (Friedman, 1979). Given these percentages and the type of information that is conveyed with non-verbal communication it is perhaps unsurprising that patients ascertain much about a practitioner without a word being spoken (Ong et al., 1995).

\section{The Philosophy of the Body and Touch}

While the current study focuses on the lived experience of touch it is worthwhile briefly examining how authors and philosophers have tended to view the concept of the body and of touch. Some authors have suggested that the traditional medical model and world-view has been based on the Cartesian view of a distinct separation between the mind and the body (Baron, 1992; Leder, 1992). ${ }^{8}$ Furthermore, Baron (1992) has argued that this traditional model views the body as a machine and that disease is a mechanical breakdown of this piece of equipment. However, some philosophers and authors have reacted against this traditional view, arguing that the body should not be viewed as divorced from the self but rather as intrinsically related to the self (Baron, 1992; Leder, 1992; Priest, 1998).

Perhaps the most well known philosopher to have discoursed on the importance of the mind-body connection is Merleau-Ponty. According to Priest (1998) "If there is a concept that gives unity to all of Merleau-Ponty's work it is that of the body qua subject: the idea that I am my body" (p. 56). While a comprehensive discussion of Merleau-Ponty's work is outside the scope of this review there are two points, underpinning his ideas about the body that are worth considering. Firstly, through the five senses we "observe" those people who we are not. Secondly, each of us is acquainted with our own body in a more direct way than by observation alone, by being

\footnotetext{
${ }^{8}$ It should be acknowledged however, that this is a traditional view and that new medical models such as the patient-centred and biopsychosocial models are a reaction against this medical paradigm.
} 
that body. Furthermore, Priest (1998) has argued that these ideas are essential to grasping the philosophy of mind of many thinkers including Hegel, Sartre, Strawson and Nagel.

The importance of the mind-body connection has also been explored in the phenomenological literature (Baron, 1992; Leder, 1992). According to Leder (1992) if one notion lay at the heart of the phenomenological view of the body it would be the idea that the lived body is an 'intending' entity. While Leder admits that the idea of intending is different for each user, for him it simply means:

it [the body] is bound up with, and directed toward, an experienced world. It is a being in relationship to that which is other: other people, other things, an environment. Moreover, in a significant sense, the lived body helps to constitute this world-as-experienced. We cannot understand the meaning or form of objects without reference to the bodily powers through which we engage them - our senses, motility, language, desires. The lived body is not just one thing in the world, but a way in which the world comes to be (p.123).

Thus it becomes apparent that from a phenomenological perspective, the body represents an individual and separates them from the world. Leder (1992) is also suggesting that the lived body is intrinsic to an understanding of the world because one cannot comprehend the environment in which we live without utilising and appreciating the senses with which we perceive it. And as has been discussed previously, one of the earliest and most primitive senses we use to engage with the world is touch.

Unfortunately, philosophical thought has not placed great emphasis on the importance of touch as a sense and thus there is a scarcity of philosophical literature exploring this sense (Nathan, 2000; Wyschogrod, 1981). Philosophers and writers have argued that touch does not lend itself to a generic theory of sense because it has idiosyncrasies that the other senses do not possess (Nathan, 2000; Priest, 1998; Wyschogrod, 1981). Wyschogrod has argued that authors who have attempted to study touch as if it were just another sense have foundered. She attempts to explain this philosophical challenge by explaining the complex nature of tactile perception: 
the body as a whole is the tactile field. The body, with its sensitivity to pressure, temperature and surface qualities, together with its kinestheses, its felt respiratory movements, its pulse, its hand's capacity for manipulative endeavour, its motility is the primordial ground of existence as incarnate (Wyschogrod, 1981, p.26).

Thus it is clear that not only is touch a unique sense, it is also fundamental to an individual's understanding of being in the world and is therefore closely allied with an individual's sense of their body.

\section{Touch and Psychology}

Another interesting area of research and clinical practice is the integration of touch into certain areas of clinical psychotherapy (Milne, 1995; Toronto, 2002). While some of the more traditional fields of psychotherapy, such as Freud's psychoanalysis, primarily relied on the verbal interchange between client and practitioner to gain intellectual insight, ${ }^{9}$ some of the more contemporary fields of psychology are adding a bodily perspective into their existing methods, either through greater awareness of a client's physical presentation or through touch (Field, 2001; Greene, 2001; Jones, 1994; Leijssen, 2006; Milne, 1995; Toronto, 2002).

Wilhelm Reich, widely regarded as the innovator of body-oriented psychotherapy (Nathan, 1999), departed from traditional psychoanalytic theory by making the therapist a more direct force in the therapeutic process. Rather than being a detached observer, the analyst sat facing the patient where they could be seen and make physical contact when and if necessary (Lowen cited in Nathan, 1999). Following these early advances made by Reich, a number of other somatic psychotherapies have developed including bioenergetics, biosynthesis, biodynamics and Gestalt therapies (Milne, 1995; Nathan, 1999).

\footnotetext{
${ }^{9}$ Currently the non-tactile view of psychoanalysis is being questioned by some authors (Toronto, 2002)
} 
In her paper Validation of the Body in Psychotherapy Leijssen (2006) describes a psychotherapeutic continuum from verbal to non-verbal forms of therapy. At one end of the spectrum are therapies that work predominantly with verbal communication while at the other end are therapies that favour body work and physical contact, usually by touch. Along with other authors Leijssen believes that appropriately used touch in psychotherapy can be both a strategic means of providing nurturance and support, encouraging openness, self-expression, and a natural expression of genuine caring (Jones, 1994; Leijssen, 2006).

\section{Touch, Culture and Healthcare}

As discussed above touch is an integral and universal component of human interaction and communication. In addition it is clear that early tactile experiences shape an individual's subsequent need for and response to touch later in life. However, implicit within the above discussion and evidenced in the literature is the idea that early tactile experiences are culturally mediated and thus the type, amount and duration of tactile experiences vary between cultures (DiBiase \& Gunnoe, 2004; Frank, 1957; McDaniel \& Andersen, 1998; Montagu, 1986; D. Williams, 1997). According to Frank (1957) “each culture fosters or specifically trains its young as children and as adolescents to develop different kinds of thresholds to tactile contacts and stimulation so that their organic, constitutional, temperamental characteristics are accentuated or reduced" (p. 241). Montague (1986) has added to this hypothesis by saying:

$\ldots$ in general, while the culture prescribes the customary socializing experiences to which the infant and child shall be exposed, idiosyncratic differences within particular families may substantially depart from the prescribed modes of behavior [sic], with more or less significant consequences for the individuals involved (Montagu, 1986, p 231).

Montagu here alludes to the fact that while vast cultural differences do exist, from a developmental perspective an important mediator of tactile experience is the early influence of the family unit. This idea is consistent with the findings of a recent study 
conducted by Helm, Kinfu, Kline and Zappile (1997) that examined the factors influencing the acquisition of a physical therapist's touching style (discussed below). The authors reported that contrary to what they had anticipated culture appeared to have little effect on the acquisition of a touching style, instead the influence of culture was found to be embedded within the context of family influences.

Recent research has examined more closely the interaction between culture and touch, often dividing cultures into high contact and non-contact cultures (DiBiase \& Gunnoe, 2004; Remland, Jones, \& Brinkman, 1995). For example Remland, Jones and Brinkman (1995) reported on an observational study which found that more touch occurred in southern European (Italian and Greek) dyads ${ }^{10}$ than northern European (English, French and Dutch). These findings were similar to DiBiase and Gunnoe's (2004) observational study which found that contact cultures such as Italian and Czechoslovakian touched more than non-contact cultures such as the American. Consistent with Frank (1957) the authors of this study concluded that this finding was most likely to be the product of socialization.

Given that our cultural background affects the way in which we communicate it is not surprising that culture has a significant impact in the healthcare setting (Ayonrinde, 2003; Goodman \& Monaghan, 2007; Seidel, Ball, Dains, \& William Benedict, 2003). According to Ayonrinde (2003) culture dictates every facet of healthcare including views on "health, illness and the search for relief from disease or distress" (p. 234). Individuals bring their own worldviews, expectations and taboos to the clinical encounter and these perspectives must be considered when a clinician engages with a patient. Finally, Ayonrinde (2003) argues that while it is not possible, in the multicultural society in which we live, to be conversant with all cultures, it is the practitioner's responsibility to be sensitive to the role culture plays in healthcare without stereotyping patients.

\footnotetext{
${ }^{10}$ Two individuals or units regarded as a pair
} 


\section{Touch and Nursing}

"Unlike medical or paramedical procedures by which the business of medicine is done, the touch of a nurse is by contrast a powerful and famous symbol of compassion" ( $p$. 95, Nathan, 1999).

At its broadest level, nursing researchers have divided touch into two categories physical touch and therapeutic touch (Gleeson \& Timmins, 2005; Routasalo, 1999). ${ }^{11}$ Although there remains controversy in the literature about the most appropriate way of sub-dividing physical touch (Gleeson \& Timmins, 2005; Routasalo, 1999) Barnett proposed a convenient division in the 1970s in which touch was divided into necessary and non-necessary touch (cited in Routasalo, 1999). This division has subsequently been used by recent nursing researchers such as Routasalo $(1996 ; 1999)$ and will be briefly discussed here. Necessary touch is generally deliberate, task oriented touch such as washing a patient or moving them in their bed while non-necessary touch is relatively spontaneous and affective, and could include a comforting pat on the shoulder. While the use and relative importance of both necessary and non-necessary touch are debated within the nursing literature (Gleeson \& Timmins, 2005), it is clear that both of these forms of touch play an integral role in nursing practice (Routasalo, 1999).

Researchers who have investigated the role and meaning of physical touch within nursing have uncovered a diverse range of themes associated with the use of touch including connection and presence (Fredriksson, 1999; Sundin \& Jansson, 2003), reassurance (Fareed, 1996; Gleeson \& Timmins, 2004; Sundin \& Jansson, 2003), spirituality (Chang, 2001; Golberg, 1998; Nussbaum, 2003) and most prominently love and caring (Bassett, 2002; Fredriksson, 1999; Kyle, 1995; Sundin \& Jansson, 2003; Van Dongen \& Elema, 2001; Wilkin \& Slevin, 2004). To summarise, Chang (2001) has attempted to define touch in nursing care as a “... behavioural process of promoting: physical and emotional comfort, appropriate social role function in caring, and sharing spirituality between people in caring situations based on humanism".

\footnotetext{
${ }^{11}$ As therapeutic touch refers to an energy technique involving no physical contact it is outside the boundaries of this review.
} 
As this discussion highlights, touch is an integral feature of nursing practice (Edwards, 1998; Gleeson \& Timmins, 2005; McCann \& McKenna, 1993; Routasalo, 1999) and yet a recent review of the nursing literature concluded that there is currently a lack of quality nursing research on the subject of touch (Gleeson \& Timmins, 2005). Gleeson \& Timmin's review identified three main areas of nursing touch research - nurses' use of touch, the impact of touch and the experience of touch. The authors also identified evidence of methodological irregularities in many of the studies reviewed including sampling bias and definitional inconsistencies in the quantitative studies as well as uncertain philosophical and methodological processes in the qualitative studies. Their conclusion was that until the current knowledge base had been extended using sound research practices the use of caring (non-necessary) touch as an intervention in itself should be resisted.

\section{Touch and the Manual Therapies}

Obviously, touch is an intimate and inseparable component of manual therapy. In fact according to the Concise Oxford English Dictionary (11 ${ }^{\text {th }}$ ed., revised) the word manual means " 1 relating to, done, or worked with the hands. 2 using or working with the hands" (Soanes \& Stevenson, 2006, p. 870). Touch is used extensively within manual therapy to assist with the therapeutic intervention, gathering information (palpation), to convey kindness or a caring message and to assist in rehabilitation (Roger et al., 2002; Talvitie, 2000). It is surprising then to find that touch is an area of manual therapy practice that has been consistently overlooked by both practitioners and researchers (Helm et al., 1997; Lederman, 2005).

Helm, Kinfu, Kline and Zappile (1997) conducted a qualitative study which examined the factors influencing physical therapists' acquisition of a touching style. The study consisted of forty interviews ( $\mathrm{n}=40)$ lasting 20 to 30 minutes and was conducted by one of four researchers all of whom were students in the Masters of Physical Therapy program at Temple University, Philadelphia. Using a convenient sampling method, clinicians were recruited from the American Physical Therapy Association Active 
Membership Profile. Using four categories identified in their conceptual framework; education, family and culture, work experience and personal-life-time experience, a non-standardised, structured interview was developed and subsequently reviewed and adjusted for content validity by a panel of six experienced physical therapists.

While the researchers explained data collection processes clearly, their account of the theoretical framework in which the data was examined was not well elucidated. Furthermore, the structured interview format (which appears in their appendix) was arranged into categories reflecting the resultant conceptual framework. The data collected were examined for common themes and a revised conceptual framework was developed. Consistent with Estabrooks and Morse's (1992) conceptual framework upon which the study was based, the paper identified three common factors which influenced the physical therapists' acquisition of a touching style - family and culture, education and clinical experience. Clinicians also identified four types of touch that they used during a treatment session. In order of descending significance these were; caring, therapeutic, "assistive" and touch used to perceive or provide information.

More recently, Roger et al. (2002) employed a more objective methodology to investigate physiotherapist's use of touch in an inpatient setting. Data collection was explained thoroughly and involved videotaping fifteen physiotherapists with three or more years experience treating two to three patients each in an inpatient clinic. Following the treatment session, the physiotherapist was asked to watch the videotape and report on the types of touch that they used. Touch definition guidelines were supplied and provided a common language to facilitate dialogue with the interviewer. The physiotherapists also participated in a semi-structured interview that consisted of six open-ended questions concerning their use of touch.

The results of the study were divided into eight single intent touch categories (categories of touch were therapeutic intervention, assistive touch, perceiving information, providing information, caring touch, building rapport, security and preparation) and thirty-three multiple intent touch categories that the physiotherapists from the present study added to the existing classes of touch. Of the single intent touch categories assistive touch was most frequently identified, followed by preparation, 
providing information, caring, therapeutic intervention, perceiving information, security and building rapport. Additionally, the interviews highlighted the role of clinical experience in the acquisition of a touching style and the importance for the physiotherapist of adapting their touch to a patient's needs based on observation. The results were verified by triangulation, peer examination, cross-case analysis, low inference data (supporting quotes were included in the study) and a participant review of data. Overall this study was a reliable and insightful investigation into the use of touch by physiotherapists and offers the reader both qualitative and quantitative data to support the conclusions drawn from the study.

Talvitie (2000) used video analysis to observe the socio-affective characteristics of the communication between physiotherapists and their patients. Talvitie found that physiotherapists used both verbal and physical guidance (through touch) more than visual guidance when interacting with rehabilitative patients. Furthermore, Talvitie described this guidance as both motivational as well as reinforcing. While Talvitie's study was not primarily looking at the types of touch used, it nonetheless provided some interesting data on the physiotherapist's use of touch. Similar to the nursing literature, this study divided touch into instructional (directing and cueing physical exercise), examination (assessing functional capacity), supportive (supporting, facilitating or resisting patients), procedural (choosing or displaying equipment) and caring (expressing empathy). Interestingly caring touch was used the least, with an average of one caring touch per session.

A more recent qualitative paper was also conducted by Ekerholt and Bergland (2006) who investigated patients' experiences of massage within Norwegian Psychomotor Physiotherapy (NPMP). According to the authors "the theory of NPMP claims that posture, respiration and muscle tension are closely related to emotional conflicts and psychological problems" (Ekerholt \& Bergland, 2004, p.403). The examination in NPMP involves a detailed case history and a bodily examination that registers and evaluates respiration, posture, function, muscles and soft tissues as well as autonomic functions and reactions. In addition the therapist considers both the patient's reaction to the examination itself and the patient's general body awareness and own self-image. The treatment in NPMP is inspired by classical massage with the therapist paying 
constant attention to the patient's respiration, using this as a guide to how the treatment proceeds. Ekerholt and Bergland's (2006) study involved ten in-depth interviews and was conducted by the primary researcher who was an experienced psychomotor physiotherapist. Based on a grounded theory approach, participant recruitment, inclusion criteria and data analysis were all clearly explained within the text and the results of the study were well supported with verbatim quotes that were labelled according to the participant and indexed into themes. Conversely, it is unclear from the paper who performed the data analysis and what quality control methods were employed to ensure rigour.

The themes that initially emerged from the data were "the ambiguity: pleasure and provocation", "the ambiguity: losing control - gaining control" and "the intra- and interpersonal dialogue". Following axial coding ${ }^{12}$ of these results two categories emerged "to be touched and to get access to own bodily information" and "interaction and mutual interpretation". A core category of "massage as interaction and source of information" also emerged. The authors concluded that their results reflect the significant impact of massage on the therapeutic process. Furthermore, they hypothesised that massage within a PMP session was a way to activate non-verbalised patterns of memories and meanings and that the patients' therapeutic journey was facilitated by sharing their experiences with the therapist through massage as well as verbal reflection. While this study provides some insight into the patient's experience of massage in Psychomotor Physiotherapy, the lack of methodological rigour limits the quality of the investigation.

\section{Touch and Osteopathy}

Osteopathy is often described as a healthcare modality that aims to examine and treat patients within a holistic framework (Parsons \& Marcer, 2006; Stone, 1999). Osteopathic authors have argued that from this perspective practitioners should

\footnotetext{
${ }^{12}$ Axial coding is the second level of coding in the analysis phase of a grounded theory study. It is a reconstructive process, which involves "categorizing, recategorizing, and condensing all first-level codes by connecting a category with its subcategories" (Polit \& Hungler, 1999, p. 696).
} 
appreciate the numerous bio-psychosocial factors that can impede an individual's recovery from ill health and attempt to identify and treat those barriers ${ }^{13}$ (Kuchera, 2005; Lucas, 2005; Parsons \& Marcer, 2006; Stone, 1999; N. H. Williams, 2007). Consistent with this bio-psychosocial approach, osteopathic authors have also argued that it is important for practitioners to be aware of the psychological impact that their verbal and physical interaction (or touch) can have on their patients and their presenting symptoms (Lederman, 2005; Nathan, 1999).

Given that osteopathic practice is a physical therapy it is not surprising that touch forms an intrinsic part of osteopathic practice. The patient is often greeted with touch (a handshake), examined with touch (palpation) and treated with touch (osteopathic manipulation). And this is just procedural touch. This does not allude to the more subtle non-procedural touch that practitioners may employ, to support and encourage a patient's rehabilitation and recovery.

While these forms of touch are an integral part of osteopathic medicine, the literature search undertaken for the current study found no research that qualifies the experience or quantifies the types of touch used by the osteopathic profession. Instead osteopathic authors have borrowed literature from other disciplines such as nursing, physiotherapy and psychology to support the strong physical, communicative and psychological functions that touch plays within an osteopathic session (Lederman, 2005; Milne, 1995; Nathan, 1999). However, given the importance of the bio-psychosocial approach and its relationship to touch it is important for osteopathic researchers to examine and critique the use and qualities of this unique and subtle facet of osteopathic care.

This chapter has outlined the broad literature base that examines the impact of touch on human experience and existence. Initially the chapter reviewed the developmental and attachment literature and its extensive role in communication. The review subsequently focused on the patient-practitioner interaction and our philosophical understanding of the body and touch. Touch was then examined in the psychological literature before the cultural underpinnings of healthcare and touch were discussed. The extensive nursing

\footnotetext{
${ }^{13}$ Or be able to refer if these barriers are outside the scope of their practice
} 
literature on touch was discussed next before concluding with the small amount of touch research in manual therapies and more specifically the osteopathic literature. The following chapter will now explore the philosophical paradigm and its practical underpinnings that guided the research process. 
Chapter Three:
Methodology 
This chapter will explore the philosophical and practical underpinnings that guided this research project. The chapter begins by briefly discussing how qualitative philosophy drives and shapes the research process and the importance of methodology to qualitative researchers. A justification for the use of a qualitative phenomenological research methodology will then follow. The chapter will conclude by examining some of the philosophical differences between Husserlian (transcendental) and Heideggerian (hermeneutic) phenomenology and by substantiating the Hermeneutic approach taken for this research.

\section{The Importance of Research Methodology}

When a researcher decides on an area of investigation or develops a research hypothesis an initial issue that requires a decision is which research methodology to employ to investigate the chosen topic (Schneider, Elliott, LoBiondo-Wood, \& Haber, 2003). The importance of the research methodology is that it influences and guides the nature of the research hypothesis or type of question that can be asked (Liamputtong \& Ezzy, 2005). In fact some authors have suggested that the research hypothesis and study design should be considered together as they reciprocally influence each other's development (Haber, 2002; Schneider et al., 2003).

Thus it is no surprise that in order to develop or design a research hypothesis or construct a research question, a researcher must have a thorough appreciation of the research paradigm within which the study is to be conducted. ${ }^{14}$ This is particularly true with respect to qualitative research since this methodology is deeply rooted in and influenced by its philosophical underpinnings (Willis, 2007).

\footnotetext{
${ }^{14}$ It should also be acknowledged however that Gadamer (cited in van Manen, 1997) believed that it was antithetical to human science research (such as qualitative) to be preoccupied with the actual method or technique.
} 


\section{Why Qualitative?}

According to Denzin and Lincoln (1998) all research is interpretive and thus is guided by beliefs and feelings about the world and how the world should be studied and understood. For example, the empirical scientists who support Cartesian dualism believe that if a phenomenon cannot be described objectively, then the significance or even existence of that phenomenon is questionable (Streubert \& Carpenter, 1999). However, both philosophers and contemporary scientists have questioned the Cartesian approach to research, particularly when research questions involve human interaction and existence. Streubert and Carpenter (1999) have said this of empirical methods "the concepts of objectivity, reduction, and manipulation, which are fundamental to empirical science, defy the authentic fiber [sic] of humans and their social interactions" (p. 3). It therefore becomes clear that some research, including research in the health sciences, requires a research paradigm that celebrates human experience and appreciates the diversity of knowing and interpretation that exists.

Liamputtong and Ezzy (2005) describe qualitative research as drawing:

on an interpretative orientation that focuses on the complex and nuanced process of the creation and maintenance of meaning. Qualitative research aims to elicit the contextualized $[\mathrm{sic}]$ nature of experience and action, and attempts to generate analyses that are detailed, 'thick', and integrative (in the sense of relating individual events and interpretations to larger meaning systems and patterns) ( $p$. 2).

From this description it becomes apparent that qualitative research is inductive and has a primary aim of interpreting information and attributing meaning. This is supported by Morse and Field (1996) who explain that qualitative research attempts to provide rich descriptions from an emic perspective ${ }^{15}$ that enables a reader to view the world through another's eyes, providing an "empathetic understanding of the world".

\footnotetext{
15 This compares to quantitative research which tests theory and causality from an etic or outsider's perspective.
} 
Within qualitative research, the researcher is a valued instrument of investigation and exploration and is thus integral to the research process. A researcher approaches the research endeavour with their own social, racial, cultural and familial history and thus has a unique perspective and philosophy about the world (Denzin \& Lincoln, 1998). These authors further identify three distinct perspectives that define the qualitative research process and guide the researcher - ontology (what is the nature of reality), epistemology (what is the relationship between the researcher and the known) and methodology (how do we gain knowledge from the world). Schneider, Elliot, LoBiondo-Wood and Haber (2003) have examined the literature regarding these three concepts and described ontology as a world view that guides the study, epistemology as providing focus for the study and methodology as providing the design for the study. Furthermore, for the qualitative researcher an acknowledgement of these influences and a critical reflection of their own beliefs, assumptions and understandings of the phenomena being researched is an intrinsic and necessary part of the research process.

There are several reasons why a qualitative research method was employed for the current research project. Firstly, qualitative research methods are commonly used when there is little information known about a subject or when an area of research is not well examined (Haber, 2002; Liamputtong \& Ezzy, 2005; Liehr \& LoBiondo-Wood, 2006; Morse \& Field, 1996; Schneider et al., 2003). This is because of the inductive nature of the qualitative investigation, whereby the researcher is attempting to construct theory rather than prove a causal relationship. Given the current scarcity of information about the use or experience of touch within an osteopathic session an explorative study using a qualitative approach was considered most appropriate for this investigation. Furthermore, authors (Cohen, 2006; Donalek \& Soldwisch, 2004) have suggested that given the inductive nature of qualitative research, it is often a useful platform or can highlight important areas of practice for empirical studies to investigate.

Morse and Field (1996) have suggested that other characteristics of the study such as the purpose of the research and the research question are also important in determining the research method. Given that this project is examining the patient's experience of touch within an osteopathic session it was deemed appropriate to use a qualitative approach that acknowledges and respects the individual's understanding and 
perspective. This position is supported by Cohen (2006) who states that "the goal of qualitative methods: to be able to see the world in the same way as those men and women undergoing the experience" (p. 132).

\section{Why Phenomenology?}

While there are some features common to all qualitative paradigms there are a number of different qualitative approaches, each attached to a unique disciplinary tradition (Polit \& Beck, 2004; Schneider et al., 2003). Furthermore, as each tradition has its own ideas about the types of questions that are important to ask it is necessary to select a method that is appropriate for the research question or problem statement that is to be researched. While ethnography attempts to explicate meaning specific to particular cultures, grounded theory seeks to describe and understand key social processes and structures. In contrast phenomenology focuses on the lived experience of individuals (Polit \& Beck, 2004).

The aim of the current study was to illuminate and explore the essential nature of touch within an osteopathic session. More specifically this study attempted to obtain findings that faithfully represented the perspectives and insights of the participants in order to explicate the essential meaning of touch within the lived experience of an osteopathic session. Thus the aims of this research were therefore consistent with a methodology and philosophy that attempts to examine the explicit nature of a phenomenon.

Van Manen (1997) states that "phenomenological research is the study of lived experience .... the world as we immediately experience it pre-reflectively rather than as we conceptualise, categorize, or reflect on it" (p. 9). Streubert and Carpenter (1999) explain that it is this lived experience that gives meaning to each person's perception of a phenomena and that an individual's experience is shaped by everything internal and external to them. Furthermore, they suggest that the goal of phenomenology is to describe this lived experience. This idea supports van Manen (1997) who described the 
objective of phenomenological research as "the description of the experiential meanings we live as we live them" (p. 11).

Phenomenological research has also been described as the study of essences (Koch, 1995; van Manen, 1997). In fact Husserl, often referred to as the father of phenomenology (Laverty, 2003), explained that "phenomenology asks for the very nature of a phenomenon, for that which makes a some "thing" what it is - and without which it could not be what it is" (p. 10, cited in van Manen, 1997). However, he also explains that an essence can only be understood or grasped through studying the phenomena as it is encountered within lived experience.

\section{Husserlian vs Heideggerian Phenomenology}

As the popularity of phenomenological research has grown, a wide range of theories have been developed and adapted from the original ideas of the most well recognised phenomenological philosophers, Husserl and Heidegger (Koch, 1995; Ray, 1994; Walters, 1995). However, Laverty (2003), Koch (1995) and Walters (1995) have all noted that confusion still exists amongst some researchers about the subtle philosophical differences between these research traditions and the importance of conducting research firmly within one of them. This section will briefly review some of the core features of these two approaches ${ }^{16}$ and in addition will discuss the use of the hermeneutic approach utilised for this project.

Laverty (2003) has argued that the key differences between the Husserlian and Heideggerian approaches to phenomenology are based around the three perspectives described above - epistemology, ontology and methodology. While Husserlian transcendental phenomenology examines the epistemological question of the nature of knowing and concentrates on the experience, Heideggerian phenomenology focuses on

\footnotetext{
${ }^{16}$ It has been noted by some authors that the phenomenological tradition should be viewed by researchers as an evolving movement rather than a discrete, static historical period of time (Laverty, 2003).
} 
the ontological question of the nature of reality and of being in the world (Koch, 1995; Laverty, 2003).

While Husserl's phenomenology was the culmination of the Cartesian tradition, Heidegger rejected Cartesianism (Koch, 1995, 1996; Walters, 1995). Husserl's philosophy involved the belief that individuals had the ability to directly grasp consciousness through intentionality (the idea that the building of knowledge starts with the appreciation of our own conscious awareness) and bracketing (the idea of identifying personal biases about a phenomenon and disconnecting from them) (Koch, 1995; Laverty, 2003). Thus Husserlian phenomenological research has been described as descriptive and focuses on the structure of experience. In contrast Hedeggerian phenomenology research is interpretive and concentrates on historical meanings of experience (Laverty, 2003). For Heidegger the idea of bracketing was impossible since the individual and experience co-constituted each other and one was therefore unable to exist without the other. Furthermore, he believed that every world interpretation is shaped by subjective, temporal, cultural and historical experiences which he called 'Dasein' (Laverty, 2003).

\section{Hermeneutic Phenomenology}

Hermeneutics is a theory that focuses on meaning and interpretation. While its early use was in the examination and interpretation of scriptural texts, it is now used to interpret and investigate human action through dialogue and text (Rice \& Ezzy, 1999). According to van Manen (1997), "phenomenology describes how one orients to lived experience, hermeneutics describes how one interprets the "texts" of life ..." (p. 4). Thus it is the interpretive element that makes hermeneutics a unique philosophical and methodological practice. Also implicit within the hermeneutic approach is the idea that there is never any truth independent of interpretation (Rice \& Ezzy, 1999).

This standpoint also affects the hermeneutic phenomenologist's research process. In a practical sense, as with Husserlian transcendental phenomenology, Heideggerian 
hermeneutic phenomenology asks the question 'What is it like?' (Koch, 1996). In contrast to the Husserlian tradition however, analysis of the data generated (often a participant interview) within the Heideggerian model is merged with the experience, assumptions and interpretations of the researcher (Rice \& Ezzy, 1999; van Manen, 1997). Thus according to Koch (1996), the subsequent interpretation and understanding of the data becomes one of unification or fusion and clearly precludes researcher objectivity from the data analysis process. Rather than denying the presence of preexisting interpretations the hermeneutic researcher attempts to reflect on how their own assumptions and understandings shape the research process.

\section{The Question of Rigour in Qualitative Research}

As with all research investigation, qualitative enquiry is concerned with standards of quality (Schneider et al., 2003). However some qualitative authors have noted that while numerous processes have been suggested to achieve academic quality, establishing a consensus among qualitative researchers on the criteria for assessing quality remains elusive (Koch, 1996; Tobin \& Begley, 2004). Considering this predicament, striving for academic quality in qualitative research is perhaps an even more important endeavour in order to maintain the value and acceptance of qualitative enquiry. This section will briefly consider some of the suggestions that have been put forward by qualitative researchers to attain academic quality and rigour.

Tobin and Begley (2004) have defined rigour as "a way of demonstrating the legitimacy of the research process" (p. 3). In the first instance Koch (1996) has indicated that a key feature of rigour within qualitative methods is the researcher's awareness of different paradigms and the way in which philosophical beliefs influence the research process. As discussed above it is imperative that the study aims are consistent with the philosophical and methodological approach taken by the researcher (Laverty, 2003).

Rigour may also be assisted if the research process is made explicit. Qualitative authors have suggested that rigorous methodological procedures are important if the research is 
to maintain its integrity and competence (Rice \& Ezzy, 1999; Sanders, 2003; Tobin \& Begley, 2004). Koch (1994) has called this process the decision trail and argues that rigour can be achieved if the reader is able to audit the events, influences and actions that guided the researcher.

There are also a number of practical activities that the researcher can employ while performing the study to increase the quality of the research. For example, various authors have discussed extensive data immersion (Sanders, 2003), utilising participant quotes to illustrate findings (Johnson, as cited in Sanders, 2003; Schneider et al., 2003), reflexivity (Lincoln \& Guba, 2000), triangulation (Janesick, 1998; Rice \& Ezzy, 1999; Schneider et al., 2003; Streubert \& Carpenter, 1999; Tobin \& Begley, 2004), the use of a field diary (Koch, 1994; Sanders, 2003) and member checking (Janesick, 2000; Tuckett, 2005) as ways of increasing the rigour of qualitative research. However, Koch (1996) has argued that due to the inherent contradictions that exist within each theoretical position within qualitative research that each research project should determine its own criteria for rigor.

This chapter has outlined the methodological and philosophical underpinnings for the current study. To begin the discussion the chapter highlighted the importance of research method and examined the broad term of qualitative research. The chapter then reviewed the phenomenological research paradigm with a brief exploration of the differences between the Husserlian and Heideggerian phenomenological traditions. The chapter concluded with an examination of hermeneutic phenomenology and a consideration of the question of rigour in qualitative research. Chapter four will now outline the practical processes that were completed to undertake the study and in addition more comprehensively discuss the methods used in the current study to achieve academic quality. 


\section{Chapter Four: Undertaking the Study}


This chapter will outline the protocols and processes used in the current study to collect and analyse the interview data. Beginning with the application and approval processes the chapter will then discuss the salient pragmatic features of conducting the interviews. A description of the theme development and the interpretation of the interview data will follow and the chapter will conclude with a brief discussion of the process that was employed in the current research to attain academic rigour.

\section{Approval Process, Ethical Considerations and Sampling}

This research was granted ethical approval on the $21^{\text {st }}$ of September 2006 by the Unitec Research Ethics Committee (UREC) for completion (of data collection) between the $20^{\text {th }}$ September 2006 and $30^{\text {th }}$ September 2007 (see Appendix A).

Given that the research involved an in-depth discussion of the participants' experience of touch, I acknowledged that potentially sensitive information could be recollected by the participant (for example, inappropriate touch). However, the risk was considered small and it was anticipated that the sampling method employed would avoid participants who would find the topic uncomfortable.

According to Rice and Ezzy (1999) qualitative research typically uses non-probability sampling since the "aim is not to generalise about the distribution of experiences or processes, but to generalise about the nature and interpretative processes involved in the experiences" (p. 42). More specifically, Morse and Field (1996) have suggested that the two main principles that guide qualitative sampling are appropriateness and adequacy. Appropriateness relates to the identification and utilisation of participants who can most accurately and succinctly inform the research in terms of the theoretical aims of the study. Consistent with this idea the current study utilised a purposive sampling technique which aims to select 'information-rich cases for in-depth study' (Rice \& Ezzy, 1999, p.43). 
The second principle of qualitative sampling is adequacy. Adequacy means that there is adequate data to develop a full and rich history of the phenomena and that no new data will emerge by conducting additional interviews (Morse \& Field, 1996). Other authors have suggested that the sample size should be determined by informational needs, whereby participants are recruited until no new information has been obtained and redundancy of data is achieved (Polit \& Beck, 2004).

For the current study three specific osteopathic clinics and osteopaths were approached to advertise and recruit for the project. Resident osteopaths were given information about the study including a description of inclusion/exclusion criteria (see Appendix B). In order to select participants who would most inform the research process, the osteopaths were asked to use their own judgement to select potential participants from their clinic who they thought would be comfortable with participation and who fulfilled the selection criteria.

Following identification, potential participants were given an information sheet (Appendix C) and a consent form (Appendix D) to consider. Participants were also given some information about my background and interest in the topic (Appendix E). Participants were then asked to contact me if they were interested in participation. ${ }^{17}$ At this stage I outlined the interview process and their involvement in the research. I also clarified any queries or concerns they had with regards to participation. Preliminary agreement for participation in the research was then obtained and a convenient time and location for the interview was organised. This is consistent with Streubert and Carpenter (1999) who have suggested that conducting interviews face-to-face at a time and place that is convenient for the participant facilitates sharing of information by the interviewee.

All participants were adults, had consented freely (both over the phone and again at the interview) and were given time between the phone conversation and the interview (approximately a week) to reconsider their participation in the research.

\footnotetext{
${ }^{17}$ Some participants requested for me to contact them directly
} 


\section{Data Gathering: The Interview}

According to van Manen (1997) the qualitative research interview serves two distinct purposes. Firstly "it may be used as a means for exploring and gathering experiential narrative material that may serve as a resource for developing a richer and deeper understanding of a human phenomenon" (p. 66) and secondly "the interview may be used as a vehicle to develop a conversational relation with a partner (interviewee) about the meaning of an experience" (p. 66). Furthermore, there are a number of different interview styles suited to the varied goals of different styles of research. Rice and Ezzy (1999) have suggested that in-depth interviews may be viewed as a continuum with structured interviews (fixed questions and forced responses) at one end and completely unstructured interviews (conversational and exploratory) at the other end with semistructured interviews in between.

At the beginning of the process I conducted a pilot interview. According to Janesick (1998) a pilot interview allows the researcher to focus on particular areas of study that were previously unclear, to test interview questions as well as to train the interviewer in establishing effective rapport and communication patterns. In the current study a pilot interviewee was chosen who had experience of both osteopathic touch as well as some experience performing interviews for qualitative research. Following the interview, the interviewee critiqued my interviewing skills and offered me feedback on style and performance. The interview and critique were digitally recorded for review and reflection.

The data for the study were collected during five face-to-face, in-depth, semistructured $^{18}$ interviews (see Appendix F) that I conducted in the participant's house. Six interviews were initially conducted and digitally recorded but the fifth interview was removed from the study because the participant did not fulfil the selection criteria. ${ }^{19}$ To ensure all relevant issues were discussed a theme list was constructed and adjusted

\footnotetext{
${ }^{18}$ While Rice and Ezzy (1999) have suggested that the term semi-structured should be avoided, as it is not consistent with a inductive approach, I have used the term to describe an interview style that uses both open and closed questions to facilitate and guide the conversation.

${ }^{19}$ It became apparent during the interview that this participant had seen their practitioner for a specific diagnosis (shoulder injury). This specific diagnosis precluded the participant from participation in the study.
} 
following each interview. In addition to recording the interviews, I took field notes that were used as a prompt at the end of the interview to ensure all avenues of data had been explored fully. The sample size in the current study was guided by the principles of recruitment listed above as well as by the number of participants that had been utilised in previous research of a similar size (Cardy, 2004; Mitchell, 2005).

I listened to each recording between interviews in an attempt to learn more about both my interviewing style and the lived experience of the phenomena. In addition, I was able to reflect on my own developing ideas about the phenomena. It was noted following the first interview that I used too many closed questions and a number of leading questions. This discovery was likely due to my inexperience, my use of a theme list and (ironically) my keenness to produce quality data. Over the subsequent interviews however I concentrated on avoiding these pitfalls and enjoyed a more exploratory and conversational style.

All interviews began with a description of the aims of the study, an explanation of the interviewee's involvement and reiteration of their right to withdraw from the interview or research. In addition participants were assured, at the start of each interview, of their anonymity and they are consequently referred to in the text by a pseudonym that reveals their gender only. This was considered significant because touch is used and interpreted differently between sexes (DiBiase \& Gunnoe, 2004). Each interview lasted approximately forty-five minutes.

\section{Transcription and Data Analysis}

Following the completion of each interview, the digital data was transferred onto a password-controlled laptop (the digital voice recorder was kept in a locked cupboard). Once all interviews had been conducted I personally transcribed the data into a Microsoft Word ${ }^{\mathrm{TM}}$ document. I considered it important to transcribe the data myself as a way of beginning the process of immersion and in addition ensuring confidentiality for the participants. Rice and Ezzy (1999) have also argued that interviewers should 
attempt to transcribe the data themselves in order to reflect on different ways of asking questions and to pick up on missed cues.

As discussed earlier the phenomenological process is an inductive one whereby themes and ideas emerge from the data. For the current study the initial process of data analysis was guided by Colaizzi's (cited in Sanders, 2003) method of data analysis.

Colaizzi's method follows seven distinct steps -

$\begin{array}{ll}\text { Step } 1 & \text { Acquiring a sense of each transcript } \\ \text { Step } 2 & \text { Extracting significant statements } \\ \text { Step } 3 & \text { Formulations of meanings } \\ \text { Step } 4 & \text { Organizing formulated meanings into clusters of themes } \\ \text { Step } 5 & \text { Exhaustively describing the investigated phenomenon } \\ \text { Step } 6 & \text { Describing the fundamental structure of the phenomenon } \\ \text { Step } 7 & \text { Returning to the participants }\end{array}$

However, as I became more familiar and comfortable with the hermeneutic approach, my data analysis was more strongly influenced by van Manen's (1997) hermeneutic perspective rather than Colaizzi's more structured process. In fact, van Manen has argued, "grasping and formulating a thematic understanding is not a rule-bound process but a free act of 'seeing' meaning" (p. 79).

According to van Manen (1997), theme analysis refers to "the process of recovering the theme or themes that are embodied and dramatized in the evolving meanings and imagery of the work" (p. 78). He also points out however, that the concept of a theme is irrelevant as it is simply a means to reach the essence of what is being researched. Van Manen argues that within phenomenological research a theme can be understood as the structures of experience. While this position is accepted, I will continue to use the word 'theme' to describe the structures of the experience that were drawn from the data. 
To gain an initial insight into the lived experience of the participants I listened to each recording four times and then read each transcript four times. As each interview was listened to and read I recorded initial thoughts and responses which occurred to me regarding the lived experience. These were noted in a research diary and formed the beginning of the analytical and reflective process.

Following the initial listening and reading, significant statements were drawn from the interviews. These statements were chosen because they appeared to best represent the participant's thoughts and feelings on the lived experience of touch. This approach is consistent with van Manen (1997) who argues that this process should examine every statement and ask the question "What does this sentence or sentence cluster reveal about the phenomenon or experience being described" (p. 93). The statements were then cut out on coloured card (a different colour to represent different participants) and arranged into initial themes on the wall under various different themes and subthemes. These themes and subthemes were then rearranged and reorganised in an attempt to most accurately represent the participant's experience. Each time a new organisation was constructed I would take a digital photograph of the thematic arrangement and their supporting statements as a way to document and review my decisions.

There was then a long period of immersion, re-engagement and reflection on the data. The hermeneutic process involves constantly engaging with the data and then stepping back. I began by re-visiting the transcripts to ensure that I had extracted all of the most significant statements. I continually reflected on the significance of the statements to the participants' lived experience of touch and how they could be understood in terms of broader themes. I then examined the statements in more detail attempting to analyse and understand their true meaning and how they fit into the wider framwork of the research. In this way I constantly challenged and tested the robustness of the emerging themes to ensure that they most faithfully represented the participants' experiences. This process is supported by van Manen (1997) who argues that 'The insight into the essence of a phenomenon involves a process of reflectively appropriating, of clarifying and of making explicit the structure of meaning of the lived experience" (p. 77). 
Throughout this process I constantly examined my own thoughts on and reflections of the meaning of the experience of touch for me. I did this by discussing the emerging themes with my research supervisors, my parents and my partner. Through this process I was challenged on the themes that were emerging and were encouraged to think of not just what the participants were saying but also what the participants were not saying.

The next phase of the analysis was the writing phase. Van Manen (1997) has argued that unlike other research which requires writing for the research report, the writing process is intrinsically connected with phenomenology and mediates a deeper reflection and action:

In an oral culture, in a society dominated by orality, phenomenology would be quite impossible. Why? Not only because phenomenology is a certain mode of reflection done traditionally by scholars who write. But also because a certain form of consciousness is required, a consciousness that is created by the act of literacy: reading and writing (p. 124).

In addition, van Manen (1997) identifies contradictions in the writing process with which the writer must come to terms with :

Writing separates us from the what we know and yet it unites us more closely with what we know ...//... writing distances us from the lifeworld, yet also draws us more closely to the lifeworld ...//... writing decontextualizes [sic] thought from practice and yet it returns thought to praxis ...//... writing abstracts our experience of the world, yet it also concretizes [sic] our understanding of the world ...//...writing objectifies thought into print and yet it subjectifies our understanding of the something that truly engages us (p. 127-129).

Through these dichotomies the researcher is able to continually question and reflect on the research process and come to a more substantial and deeper understanding of the lived experience. 
Thus through an exhaustive process of writing and re-writing the developing themes I was able to continue to build and reflect on my initial analysis and interpretation of the data. Moreover, through the writing process I was able to more fully understand and appreciate the interweaving and inter-dependence of each of the themes and how they contributed to the phenomena. In addition, I began to sense how the themes were bringing me closer to a deeper and more comprehensive understanding of the phenomenon of touch within an osteopathic session.

\section{Maintaining Rigour}

While there is little consensus amongst qualitative researchers on the best way to assess academic rigour, it is still an important goal for qualitative researchers to strive for. In keeping with this spirit, this section will briefly outline the steps taken in the current study to achieve academic quality.

According to Lincoln and Guba (2000) reflexivity is the process of critically reflecting on the self as a researcher. They argue that it is the process of coming to terms with our selves regarding the way in which our research is shaped and founded on the "binaries, contradictions and paradoxes that form our life" (p. 183). Schneider et al. (2003) support this by suggesting that the researcher should be conscious of the fact that they are a product of a particular social scene which invariably affects the way in which phenomena are observed.

Before data collection began I examined my own preconceptions about the topic by having another qualitative researcher interview me utilising the same interview schedule that I was to use throughout the study. To confront my own bias, the ideas from the interview were regularly reflected on throughout the analytical process to identify how my own history and understanding was influencing the process. I also attempted to maintain open to the participants' experience throughout both the interviewing and the data analysis phases. This involved constantly reflecting on my thought processes and attempting to understand how these processes were influencing the themes that were 
developing. As part of a reflexive technique, I have also included a brief summary of my history and my processes to arrive at the research topic in chapter one.

Triangulation techniques were also used to increase the rigour of the study. According to Denzin (cited in Janesick, 1998) there are four basic types of triangulation -

1. data triangulation which uses a variety of data sources

2. investigator triangulation which uses several different researchers or investigators

3. theory triangulation which uses multiple perspectives to interpret a single set of data

4. methodological triangulation which uses multiple methods to study a single problem.

In the current study, I used a combination of investigator triangulation (by consulting my supervisor and family members in the analysis process) and theory triangulation (by re-engaging with the data and re-examining developing themes within a number of different fields of study).

As discussed earlier another way of increasing the rigour of a study is to keep a field diary so that the decision trail can be followed (Koch, 1994; Sanders, 2003). For the duration of the study I documented all experiences, thoughts and insights that occurred to me about the research topic. Furthermore, I thoroughly documented my analytical process as I listened and read the transcripts during theme development. This process allowed me to reflect more accurately on previous themes and ideas that had occurred to me. Finally, the analysis of themes in the following chapter includes verbatim quotes from the participants to ground the themes more firmly in the data and to increase the rigour of the analysis. 


\section{Introduction to the Study's Participants}

As discussed, five participants were involved in the research process and their experiences provided the basis for the themes generated in chapter five (data analysis). To ensure their confidentiality all participants were given a pseudonym, which indicates their gender only. As has been discussed, it was considered important to retain the participant's gender for the reader as the experience of touch is mediated by an individual's gender.

$\begin{array}{clll}\text { Interview } 1 & \text { Participant } 1 & \text { Wendy } & \text { (female) } \\ \text { Interview } 2 & \text { Participant } 2 & \text { Jennifer } & \text { (female) } \\ \text { Interview } 3 & \text { Participant } 3 & \text { John } & \text { (male) } \\ \text { Interview } 4 & \text { Participant } 4 & \text { Sarah } & \text { (female) } \\ \text { Interview } 5^{20} & \text { Participant did not fit selection criteria } \\ \text { Interview } 6 & \text { Participant } 5 & \text { Phil } & \text { (male) }\end{array}$

\section{A Guide to Reading Chapter Five}

The following chapter provides a discursive exploration of the themes that emerged from the participant's interviews about their lived experience of touch. The chapter is organized into three major themes with a brief discussion of how each theme is represented in the data. Each major theme is represented by a letter of the alphabet in square brackets - $[\mathrm{A}],[\mathrm{B}]$ and $[\mathrm{C}]$.

The three subthemes that follow then explore various aspects of the major themes in more detail. Each subtheme is indicated with square brackets and is typed in italics.

\footnotetext{
${ }^{20}$ As explained in this chapter the fifth interview had to be abandoned because it was realised that the participant did not fit the selection criteria.
} 
Each subtheme is also numbered under each major theme, for example - [A1] Engagement - let the session begin, [B2] Trust - a prerequisite for physical contact or [C3] Confidence - very reassuring.

All participant quotes are indented and italicised to distinguish them from the researcher's analysis and interpretation. In addition each quote has been referenced with the participant's pseudonym and transcript page number.

Symbols used in the text are as follows -

$\begin{array}{ll}\ldots & \text { Pause in the dialogue } \\ \ldots / / \ldots & \text { Omission of dialogue } \\ {[\text { Text }]} & \text { Text is added or altered for clarification }\end{array}$

This chapter has outlined the protocols and processes that were followed in the completion of this research project. It began by describing the application and approval processes and then summarised the process of participant selection, data collection and transcription. This was followed by an exploration of the data analysis phase of the research and the processes that I engaged in to ensure a high level of rigour throughout the study. The chapter concluded with a brief introduction to the participants and a guide to reading the next chapter. In essence the aim of this chapter was to provide an insightful backdrop to the following chapter, which analyses the participants' lived experience of touch in an osteopathic session. 
Chapter Five: Data Analysis 
This chapter represents the culmination of the thematic analysis as described above. The participant's lived experience of touch within a osteopathic session involves an intricate and complex communicative interaction between themselves and the practitioner. In exploring this interaction the study revealed three main themes: [A] The Process - a physical interaction between the participant and the practitioner, [B] Professionalism - the practitioner's responsibility and [C] Reassurance - a therapeutic necessity. In the following discussion each of these themes will be broken down further into three subthemes in an effort to simplify the data and best represent the participant's experience.

The chapter is organised into the three major themes with a brief description of how each theme is represented in the data. Each major theme will begin with a quote from one of the participants in an attempt to engage the reader with the 'flavour' of the theme and ground the theme in the data. The three subthemes that follow will then explore various aspects of the major theme in more detail. The chapter will finish by summarizing the themes in an attempt to elicit the fundamental structure of the phenomenon.

\section{THEME [A]: The Process - a physical interaction}

Yes, the information is given verbally. But the dialogue is also a voiceless one, a silent one of interaction with you as a physical object, as a personas a physical body. The way he approaches you, the way he touches you, the way you are dealt with. You end up feeling integrated, a whole, respected, and it is a very satisfying or very rewarding process on its own (John, p. 9).

Theme [A] draws attention to the importance of the physical patient-practitioner relationship in the therapeutic process. Essentially, it relates to the way in which the 
practitioner physically engages with the participant's body and the physical and mental consequences of this interaction. Subtheme [A1] Engagement-let the session begin, initiates the interaction between the client and the practitioner and indicates to the patient that the practitioner is attuned to their body. Subtheme [A2] Dialogue knowing hands converse with an expressive body, acknowledges the creation of a distinctive reciprocal interaction between the practitioner's hands and the participant's body that is central to the healing process. Finally, subtheme [A3] Support - just holding, establishes the practitioner as the foundation on which the patient's health will be nurtured.

\section{[A1] Engagement - let the session begin}

Engagement is the process by which the practitioner makes the first physical contact with the participant's body. Normally, this action is initiated near the start of the session and will usually continue throughout the entire interaction. This process is one of acknowledgement and often has a gauging quality whereby the participant is aware of the practitioner sensing their disposition. The process may begin with some nonprocedural touch such as a handshake or with some procedural touch whereby the practitioner guides the patient through a range of different movements or when the practitioner palpates the patient's spine. However, the central feature of this touch for many of the participants is that it indicates the session has begun and the practitioner is engaging with their body.

John identified the moment his practitioner first made contact with him in the waiting room and interpreted this as the practitioner's way of gauging the physical and psychological disposition of his patients:

The practitioner will touch you on your shoulder, an arm around the shoulder, but its very circumspect. He is obviously at this stage, picking up your vibe. As you are approaching he is trying to work out where I am at, 
and understand what is appropriate in that initial period of ... getting used to what my state is (John, p. 1)

He then describes the first physical contact that occurs in the treatment room and describes this as another information gathering exercise on the part of the practitioner:

This is the first therapeutic touch. Not therapeutic, it's diagnostic. He is feeling what is going on, and asking me to make movements, like bending forward, touching your toes, lift one side. Trying to get some sense of what is happening in the spine (John, p. 2).

The importance of this statement is that John not only appears to be conscious of when the touch first occurred but also that he is aware of the practitioner attempting to achieve something with these actions. This is significant because it indicates to John that the practitioner is touching him for a specific purpose and is thus engaged with his body. In fact subsequent to this statement John indicates that this initial touch signifies the start of the therapeutic process and that he is aware that the practitioner has engaged with his body and that he is part of this process:

You feel as though he is paying attention and that you are ... yeah ... he is involved, you are starting to be drawn into the process of what is about to happen (John, p. 2).

While Phil describes a slightly different routine he also identifies touch as a significant feature of the diagnostic process early in the session:

And then he gets down to business pretty quickly, he asks me to stand up in front of him, so he can look at me. Gets me to bend in various directions to assess ... (Phil, p. 2)

I think it was once I had gotten onto the bed, onto the treatment couch he showed me how, and where he wanted me sitting and then he starts to have a look and examine your back and so on ... (Phil, p. 2). 
He sort of ran his hand down gently looking at, feeling spots that he had obviously looked at before (Phil, p. 2).

In his example, Phil explains that his practitioner initially asks him to make various movements before making physical contact with his body to examine more specifically areas of interest. Like John, Phil identifies this as the start of the session, and is aware of the practitioner's focused attention on areas of interest when he physically touches him. Phil's use of the pronoun "your" in the first quote is also interesting. It suggests that Phil believes the touch he is receiving is similar to that which is given to all other patients. Thus for Phil it could be reasoned that this type of touch is intrinsic to the osteopathic process.

When asked if she had any advice she would like to give osteopathic practitioners Wendy made a direct link between the importance of the practitioner's attention and the way that this is reflected in their hands:

You know, being interested in your subject, you know... believing in what you are doing and I am sure that that kind of... reflects in how you work through your hands (Wendy, p. 8).

\section{[A2] Dialogue - knowing hands converse with an expressive body}

This subtheme relates to a physical conversation that occurs between the practitioner's hands and the participant's body. Many of the participants alluded to this theme and it appeared to manifest for them in two distinct ways. While the practitioner's diagnostic and treatment goals were communicated to the patient through their hands, the participants were aware of the information that their own bodies communicated back to the practitioner. For the participants this unspoken dialogue between themselves and the practitioner underscored a reciprocity that is characteristic of the patient-practitioner interaction and is pivotal to the therapeutic process. 
While all of the participants referred to the physical dialogue some participants referred to it directly whereas others alluded to an unspoken interpretive touch that the practitioner used for diagnostic purposes. Of those who referred to it directly John expressed this about some of the initial touch from his practitioner:

I imagine he is making diagnostic interpretations. Asking me questions like "is it stiff?"... "does it feel easy?" So you are in a dialogue with ... touch is definitely part of the deal because he is feeling and making interpretations (John, p. 13)

In this statement, John explains that for him touch is part of a greater dialogue between the patient and the practitioner that includes both physical and verbal communication. This description of the practitioner's actions and instructions illustrates John's interpretation of their motives and thus represents a conscious perception of the dialogue that has been created between them. John also alludes to the practitioner reading his body or attempting to elicit information from his body that will assist with the evaluative process and thus he appreciates his own involvement in the dialogue.

This diagnostic or interpretive touch described by John was also experienced by Sarah who said this of her practitioner's touch:

He would normally assess me before we do any work on the table. So I will normally stand up and face the wall and he will kind of feel along my vertebra, my hips, my neck, maybe my shoulders ... (Sarah, p. 1).

When asked about her interpretation of this particular touch she replied:

Well I think he is trying to work out where the problem areas are and what areas to work on (Sarah, p. 2).

The participants also noted the responsive quality to their practitioner's touch. Rather than just being routinely examined and treated, they felt that their practitioners listened to their bodies and modified their touch to suit their individual condition. 
Wendy put this simply:

... so I guess really my treatment is adapted to where my body is at (Wendy, p. 5).

John also experienced this responsive quality in his practitioner's touch and felt that this quality was common to osteopathic practitioners:

Their [physiotherapists] training is such that they have this technique, they do it and the idea that there is some sense of a dialogue is involved is just not part of their training. You are supposed to respond to what the technique is, whereas when I am with an osteopath there is a sensation is that it may not work and it is not my fault, it needs some how ... there is a responsive quality to osteopathy which I don't get from traditional medicine if you can call it that (John, p. 8).

In this quote John again hints at this idea that touch is a reciprocal process that involves both the practitioner and the patient's response to treatment. This understanding is supported by a comment which John had made earlier in the interview about his feeling that he wanted to be able to convey through his body as much information as he could to the practitioner about his condition:

You are starting to be drawn into the process of what is about to happen, it's interpretive and I am trying to give him the best information that I possibly can so he can give me the best service that he can give me. Because I am giving him the best understanding that I can generate to give him enough to work on (John, p. 2).

\section{[A3] Support - just holding}

The idea of support and its importance to the therapeutic process was present throughout all of the transcripts. The participants articulated this idea of tactile support 
in different ways. While most participants expressed the view that their practitioner used a generally supportive approach when interacting with their bodies some also described specific techniques that induced a sense of being supported. For most of the participants this idea of support had both physical and psychological components and translated directly into a feeling of being cared for by their practitioner (see also subtheme [B1]).

This supportive facet of the practitioner's touch was expressed by the participants in a number of different ways throughout their interviews. John explained that for him it was the practitioner's hands or the way in which the practitioner held him that somehow induced a sense of support or acceptance within him:

Well, it's just I am a sucker for touch. The business of having someone have their hands underneath ... and he is letting you weigh down, he is wanting you to relax into his hands. That sense of what you are doing is acceptable is nice to feel. What you are doing is ... he wants you to relax into it ... // ... what you are doing is acceptable, you don't have to do any presentation, you just need to relax and it nice to have somebody let yourself ... put yourself into their hands (John, p. 3).

Clearly, for John one of the most enjoyable features of his sessions with the osteopath is that he does not have to put on any act for the osteopath, that he is able to be completely himself. In fact John believes that this is not only what the practitioner expects from him as the patient but it is what they want. John also describes a neutral quality to his practitioner touch whereby there is no input into his system but rather the practitioner is just there with him. Thus there is a dialogue, but one that is completely lacking in practitioner judgment. In addition this neutral touch is comforting for the simple fact that it holds him:

The feeling of touch is comforting, but it is not ... the energy is not flowing into my body it is just holding it (John, p. 10). 
And the feeling with my osteopathic practitioner is that it [touch] is neutral. And this may be crucial to the whole attitude you have to touching a client ... // ... that it is really possible to add energy, and energize somebody or take it away. People can suck you dry as it were, but there is this neutral, comforting, non-interfering type of touch (John, p. 10).

John further explains that the presence of this support throughout the session becomes patently obvious at the end of the session because the osteopath withdraws his support in order to force him to become "self-responsible" again:

But right at the end of the session when it was happening you sort of, you do have this sense of being dropped. That he has tried his damndest and you really haven't co-operated and it is kind of your fault, and its as much as he can do, and you will have to put up with it from now on. So there is a sensation of having to re-establish my centre and become self-responsible again. A curious sense of being shunted out of the nest (John, p. 8).

This quote illustrates the support that John feels on two different levels. Firstly, the use of the word "dropped" in this statement supports the idea that the practitioner is somehow physically supporting John in this therapeutic process. Secondly, John refers to having to "re-establish my centre and become self-responsible again" which suggests that his psychological state is also being tended to. In addition John's reference to "being shunted out of the nest" supports the idea that he is forced to become selfresponsible again (as a child might have to).

Wendy also expressed this supportive element by comparing her practitioner's touch with another physical therapist who had previously treated her. Wendy explained that her practitioner's tactile intentions are expressed:

In a kind of supportive way. I don't feel it ... it is not intrusive. I think that is what I found difficult with that massage was that it was a bit . . you know she was obviously very keen, her intentions were good. But it was a bit too intrusive (Wendy, p. 5). 
Thus it can be seen from this statement that one of the qualities Wendy appreciates about her practitioner is that they are physically with her, without being invasive in their presence. There is a sense of the practitioner being present without overloading Wendy's system.

Phil compared this holding element with the sort of touch you might receive while being cared for in hospital:

I guess it is the same sort touch that you get when you are in hospital and you are getting sponge bathed. You sort of feel relaxed and comfortable and nurtured I guess ... I think that is the word. Yes, it is sort of nurturing and you are sort of ... you know that there is a certain calmness about it (Phil, p. 7).

This comparison is particularly interesting as a patient's condition and medical needs in hospital are often completely different from when seeing an osteopath and yet Phil has identified a common descriptor for the types of touch that one receives in both of these settings - a nurturing touch. According to the Concise Oxford English Dictionary $\left(11^{\text {th }}\right.$ ed., revised) the word nurture comes from the Latin word nutrire to nourish and defines it as " 1 rear and encourage the development of (a child). 2 cherish (a hope, belief, or ambition)" (Soanes \& Stevenson, 2006, p. 982). This definition is consistent with both Wendy and John's descriptions above and in addition hints at a latent characteristic of this touch or perhaps makes reference to a primitive need for this type of touch.

\section{THEME [B]: Professionalism - a practitioner's responsibility}

For me personally, it is not an issue and I look for and want [touch] to be a natural component. If I feel that, if my osteopathic practitioner were to be embarrassed by the feeling of the contact that is being developed, I would then have a problem with him, because I need him to be easy so that the 
process of touch is just second nature. It is just like the sun rising and the sun setting. It is part of the deal. If someone is "sensitive" and aware that there could be a problem, then it does become a problem. You start, fulfilling the prophecy as it were (John, p. 6).

Theme [B] highlights the role of touch in developing professional boundaries and emphasises patient expectations surrounding the physical contact which occurs in an osteopathic session. Subtheme [B1] Care - an empathic quality examines how the practitioner's touch creates a relaxing sense of being looked after within the participants while subtheme [B2] Trust - a prerequisite for physical contact examines how touch establishes a sense of trust between the participant and the practitioner. To complete the professionalism theme, subtheme [B3] Boundaries - a professional relationship continues on from [B2] by exploring how touch helps establish professional borders in which the therapeutic relationship exists. All three subthemes acknowledge the importance of other elements of communication, for example verbal dialogue, however because of the intrinsic role touch plays in client-practitioner communication it is deemed to have a pivotal role in developing the professional relationship.

\section{[B1] Care - an empathic quality}

Most of the participants also discussed the issue of care within the therapeutic relationship. While not all of these participants directly articulated a link between their sense of being cared for and the way in which they were touched by their practitioner, it was obvious from the transcripts that a sense of care and support was mediated by both a verbal and physical dialogue. In addition, some of the interviews revealed a sense that this feeling of being cared for relaxed the participant. For some participants this sense of tactile care was created through the practitioner's understanding of their bodies while other participants were able to specifically articulate experiencing a tenderness in the practitioner's handling which conveyed a sense of care. There was also a common association between the feeling of care and their practitioner's empathic approach. 
Sarah described this of her practitioner's touch:

You know if he finds something is really locked up, he will be like "oh god, no wonder you weren't feeling good". It just gives you that kind of feeling of he knows where you have been or what your body has been going through. It does make you feel cared for ...//... and I guess he is happy to be, or seems to be happy to be someone who does that for people or can make them feel that (Sarah, p. 6).

From this statement it becomes apparent that her practitioner's palpatory skills as well as their understanding of "where you have been" gives an empathic quality to her practitioner's touch. More specifically it becomes clear that this empathic element of the touch is mediated by the way in which Sarah's practitioner employed techniques and concurrently listened to what her body was telling him. This statement also lends itself to the idea that it is an interplay between the verbal and tactile communication that creates this feeling of care. John also made reference to feeling cared for when he described the way in which his practitioner handled him:

There is a lovely sense of trust that I have eventually managed to put together with [my practitioner] where you just kind of know that he is going to look after you (John, p. 5).

As already mentioned some of the participants also described specific techniques that engendered a sense of support or being cared for. Interestingly two of the participants described techniques that involved the practitioner holding their head as particularly relaxing and caring. Wendy identified the moment that her practitioner began some cranial work on her :

I mean the minute she put her hands on my head I felt, my brain felt more or my head felt more soothed (Wendy, $p$. 7)

John also identified a relaxing feeling associated with having his head touched and held: 
Once again it is relatively pleasant to have someone cradle your head. It's a beautiful thing. It may be satisfying some diagnostic process but it also makes you feel at one. It's a nice feeling (John, p. 3).

Phil described the care that his practitioner displayed toward him within the broader context of the osteopathic approach. He said this of the techniques that his osteopath used:

There is an enormous sort of gentleness in the approach which I guess is characteristic of osteopathy that is very reassuring and it makes you probably relax all of your major musculature so that you are actually easier to treat you know. [And] of course you don't feel any surrounding soreness afterwards (Phil, p. 8).

Two things are significant about this statement, firstly the use of the word approach suggests that touch is a component of care rather than a single contributing factor and in addition suggests that the lack of residual pain following the treatment also reinforces this sense of care.

For Wendy this caring touch was best described by comparing her practitioner's touch with that of a nurse she had seen earlier in the day:

I think in their fields they were probably on a par. They were both wanting to help and do a good job, you know. And there was obviously a kind of tenderness or compassion [to their touch] (Wendy, p. 7).

This comparison is significant because nurses are often a symbol for care and trust within healthcare, and because the participant had clearly experienced these same qualities in her relationship with her osteopath. In addition, when asked how she felt when her practitioner put her hands on her she replied: 
I suppose I would say probably restored. You know, I suppose it is almost like I have been giving out a lot so its good to be you know, be given some input myself. You know healing (Wendy, p. 6).

\section{[B2] Trust - a prerequisite for physical contact}

Subtheme [B2] Trust - a prerequisite for physical contact attempts to explore more deeply how the practitioner's touch contributes to or mediates a trusting relationship between the participant and their practitioner. All of the participants had a substantial amount of trust in their practitioners and in fact, as would be expected, it was a prerequisite for continuing to see them. While no firm conclusions can be drawn about the relative contribution of touch in developing this sense of trust, it became apparent from the data that it was a product of both the verbal and physical dialogue that took place between the practitioner and the participant that generated this sense of trust. In general trust in the practitioner's hands was a process constructed and negotiated over time.

For most of the participants the trust that developed through touch was closely allied with their perception of the competence and confidence of their practitioner. All of the participants felt that their practitioners were competent and this was mediated through the way in which the practitioner handled them, that is through their touch. Sarah explained the type of touch that she felt promoted a sense of trust in for her:

I guess that my osteopathic practitioner is in the bracket of all the other people that I have had, that seem to know what they are doing and are comfortable with it and don't usually make a muck up and don't get flustered or sweaty hands or things like that (Sarah, p. 8).

The idea of the practitioner being comfortable with the touch, and the sense of trust that this promoted, was also alluded to by John who said: 
So, my view of all such touch is that it has got to be direct, easy, accepted and thereby becomes non-remarkable. It just is. It just happens. I am aware of it, I like it, it's not a problem that it happens, I want it to happen, but at the same time it must not be, it's just there (John, p. 7).

The trust that some of the participants had in their practitioner's touch was also evident in their ability to completely put themselves in their practitioner's hands. Wendy explained this simply:

I hand my body over I suppose you would say (Wendy, p. 3).

Some of the participants also discussed the impact of previous experiences and notions about the appropriateness of the touch and how this related to trust. John for example explained that he had had a substantial amount of psychotherapy that involved a lot of bodywork with a male therapist. In particular he highlighted the potential for the intimacy of the act of touching to be an issue in the therapeutic relationship although for John this was not a concern:

Male to male closeness in this culture is very problematic. And the issue is obviously that men will find that difficult to cope with. For me it's never been a problem (John, p. 5).

I am personally totally aware of the therapeutic nature of it [touch], and I enjoy the closeness to another human being, because it is another human being in this kind of intimate contact. But it doesn't threaten me. I am not threatened by it (John, p. 6).

While all participants alluded to the relationship between trust and their practitioner's care it was John who best summarized how trust is developed in a practitioner's touch:

And it [touch] is everything because it is wordless. You can't lie with your body very easily. If your body is doing something, it's because ... unless you 
are an incredibly good actor, it's because you really do mean it (John, p. 12).

\section{[B3] Boundaries - a professional relationship}

Theme [B3] Boundaries - a professional relationship relates to the way in which touch mediates some of the professional boundaries that exist within the patient practitioner relationship. For the participants in this study touch was intrinsically connected to the creation and maintenance of a clinical distance that should be honoured between the patient and the practitioner. The idea of maintaining boundaries was regularly linked to the practitioner's professionalism.

The issue of how touch influenced the boundaries of the therapeutic relationship was alluded to in all of the interviews. Interestingly while the participants acknowledged the emotional or psychological impact of the physical contact that they received from their practitioner, they were also clear that the tactile contact from the practitioner should be entirely neutral in terms of practitioner emotion. It was also noted that the majority of participants described procedural touch (i.e. aimed at either diagnosis or treatment) with only some participants alluding to non-procedural touch. When asked about this discrepancy the participants felt that non-procedural touch added an intimacy to the interaction that they were not comfortable with.

Wendy attempted to illustrate the neutral element of her practitioner's touch by comparing the touch she received from her practitioner with that which she received from a close family member:

I would say it is different because it is professional isn't it. I mean my mum has massaged me and I think that is different. I mean she was on a massage course and she was training. I think that it is different ...//... because you have got that emotional link, haven't you? ...//... like with a family member. 
There is that emotional link, whereas when you go to see a professional it is different (Wendy, p. 7-8).

John was more direct in his assessment of the patient-practitioner relationship:

There is no intention behind my osteopathic practitioner doing anything, in terms of establishing an emotional relationship; [he] is very clear about the limits around that. I am really aware of what he would do or could do to ... increase the temperature if you like of the interaction between us (John, p. 7).

John's indicates here that he is aware of the potential for an emotional connection to be developed through touch but is equally conscious that his practitioner avoids this and in fact establishes boundaries around the interaction. He also offered this piece of advice for physical therapists:

[Health] Professionals have been given the licence to touch you [and] that you need to never transgress that license in order to maintain your relationship to your client which is fear free (John, p. 11).

In this quote John speaks of a licence issued to health professionals that allows them to touch patients. Implicit within John's statement however is an understanding that licenses have clear restrictions and boundaries within which they may be used and these should be known and respected by the license holder. John alludes to a link between infringing on the conditions of this licence and feeling unsafe.

Boundaries within the professional relationship were also discussed in relation to the use of procedural and non-procedural touch. As mentioned earlier the participants appeared divided on the presence and necessity of non-procedural touch. It was noted that most of the participants neither reported nor required non-procedural touch from their practitioner. In fact for these participants the absence of non-procedural touch added to the professionalism of the practitioner. When asked about her practitioner's use of non-procedural touch Jennifer expressed this view: 
I think that the touch with the purpose in the treatment is really important of course, but other than that ... Maybe shaking hands on first meeting but other than that I don't think much else is appropriate really (Jennifer, p. 8).

Jennifer admitted that while she enjoyed a lot of touch with friends and family in her in her personal life she had clear ideas about the appropriateness of touch in a professional setting:

As a counselling professional I have clear ideas about what I would professionally do. Say when I am visiting people at an old age home I probably wouldn't touch unless invited. But I know that some people really like a hug, or a touch and others don't. And until you get to know people you just don't. I mean I have quite clear ideas about what's appropriate. So my stage isn't touchy feely. In a professional setting that's the way (Jennifer, p. 7).

In this statement Jennifer acknowledged that non-procedural touch (such as a hug) is an individual preference but that she personally has clear ideas about the types of touch she engages in and is willing to engage in a professional setting.

In contrast other participants reported the use of non-procedural touch by their practitioner particularly as a way of beginning or ending the session with very little nonprocedural touch reported throughout the session (see subtheme [A1] Engagement - let the session begin and subtheme [C2] Competence - a knowing touch for examples of non-procedural touch). Participants also acknowledged a deliberate and gauging element to the non-procedural touch when it occurred, which for them imparted a sense of professionalism.

What also became apparent from the interviews was that each participant had strong feelings about the importance of non-procedural touch in establishing boundaries in the therapeutic relationship. Sarah had this advice for osteopathic practitioners with regards to non-procedural touch: 
I think, and as a natural health practitioner myself, you have got to find your own way with things. And your own style of touch will come out but you have just got to always use your intuition to what the client is wanting, where the client is at. If they are being very closed kind of "blah blah" people, then they probably don't want a pat on the shoulder from you in the first session (Sarah, p. 9)

Sarah's views were supported by John, who believed that while the boundaries of touch within the session were negotiable practitioners had to be sensitive to the patient's needs and limitations:

The fear of touch and the implications of it, but the realization of the appropriateness is definitely part of the mitigating quality. That when you are with a person there is just so much that they can deal with, so you have to be sensitive about what it is that you are doing. That they can't or can cope with. But always to be able to; its as though you go to the limits of the client and the client controls, but if the client is more relaxed you can go to their limit, so that your limits are always bigger than the limits the client has given you. But that requires courage because at some point you are going to get to the position where you hit the wall, your own wall (John, p. 13).

John is clearly aware of the reciprocal and flexible nature of the boundaries. While he believes the establishing of clear boundaries requires courage, he is also clear that it is the practitioner's responsibility to manage this negotiation and that it should ultimately be the patient's boundaries that dictate the extent of the interaction. 


\section{THEME [C] - Reassurance: a therapeutic necessity}

Then he moved onto the head and he gave a lovely motion that you guys must get confident. I mean I don't know how you get confident, you hold the head and then kind of loosely move it. And ask me to breath in and then relax and you know it is coming. A jolt and he clicks it. Once again, a beautiful sensation (John, p. 5).

Theme [C] discusses the role of touch in reassuring the patient about both the skill of the practitioner and the nature of their condition. The role of reassurance spans both interpretive and treatment touch and gives the practitioner credibility in the eye of the participant and is a necessary component in the therapeutic process. Subtheme [C1] Knowledge - with seeing fingers examines how the practitioner's training and expertise are exhibited through the way in which they touch the participant. Subtheme [C2] Competence - a knowing touch explores the idea that touch is a transparent act which allows the participant to assess the practitioner's professional aptitude. Closely linked with this idea, subtheme [C3] Confidence - very reassuring examines how the practitioner's approach to the physical interaction reassures the patient of their competence. As with theme [B] Boundaries all three subthemes are supported by a verbal dialogue and in addition contribute toward reassuring the patient about their condition and the possibility of some resolution.

\section{[C1] Knowledge - with seeing fingers}

Subtheme [C1] explores the way in which the practitioner's hands conveyed a sense of the practitioner's detailed anatomical knowledge. Moreover, the practitioner's informed and responsive touch imparted a sense of academic and practical competence to the participant that was very reassuring. 
What many of the participants said about their practitioner's touch was that it felt as though the practitioner knew what they were touching. Jennifer compared her practitioner to a General Practitioner in an attempt to define her practitioner's touch:

I just feel that [my osteopathic practitioner] feels more about the muscles and bones and joints than my GP would. I feel as if she knows what she is touching. Well, I mean I guess doctors do to but not so much in the muscle and joints and things (Jennifer, p. 6).

This observation suggests that one of the reasons Jennifer visits her osteopath is that she believes she has a comprehensive anatomical knowledge of the musculoskeletal system. Moreover it is clear that Jennifer believes her practitioner has a practical knowledge which allows her to know what she is touching when she puts her hands on her body.

Sarah also talked about the quality of her practitioner's touch and alluded to her practitioner's knowledge of the musculoskeletal system:

I mean, yeah it is usually quite a firm pressure, you know you can feel him feeling the joints, and the muscles as well, its more of a pressing touch than a rubbing, stroking action (Sarah, p. 2).

This quote also suggests Sarah is aware of the interpretive nature of her practitioner's touch as well as the information her practitioner is able to extract from her body. John was also conscious of his practitioner's anatomical knowledge and made a comparison to his experience of physiotherapists:

There is a process that they [physiotherapist] put you through and you feel very much more on a production line than you do with an osteopath, where you have a sense of the particular structure is being taken into account and you feel much more individually treated than you do with a physiotherapist who regards it as your problem, you have to do the exercises and its your issue (John, p. 8). 
John's reflections here highlight two significant points related to his practitioner's touch. Firstly, John is aware of what his practitioner is doing; he is conscious of the practitioner palpating a particular "structure". Secondly, he is aware of the responsive quality of the practitioner's touch, whereby the practitioner is treating and adapting their diagnosis or treatment in response to what is being palpated. As mentioned earlier, this responsive quality also enhances the perception of a physical dialogue between the practitioner's hands and the participant's body.

Phil also alluded to this ongoing dialogue and the practitioner's purpose for the touch. In particular, Phil appreciates that the interpretation of his body is done efficiently and utilizes both visual and tactile senses:

He sort of ran his hand down gently looking at, feeling spots that he had obviously looked at before. He is pretty quick to, he goes directly to the area (Phil, p. 2).

I think he is just confirming with touch what he had seen already. It's very light and sort of cursory (Phil, p. 2).

Phil also compared the interpretive nature of his practitioner's touch to his own experience as a teacher:

But the other thing, that struck me as quite extraordinary is that he seems to see with his fingers. I am used to seeing with my ears as a singing teacher but he almost unerringly puts his finger in exactly the right spot to find sort of where things are hot so to speak. He doesn't do a lot of fumbling around to find it. He already knows where he is. And that is quite reassuring (Phil, p. 5).

This last point is a consistent feature across the interviews, that is, the ability of the participants' practitioners to identify very quickly through touch an area significant to 
them. This reassured the participant about both the practitioner's competence (see subtheme [C2]) as well as their own experience of their bodies and their condition.

\section{[C2] Competence - a knowing touch}

This subtheme draws attention to a quality of the practitioner's touch which generated in the participant an appreciation of the practitioner's professional skill. Closely linked with subtheme [B2] trust, it appeared that practitioner competence was a major determining factor in choosing to see a particular osteopath. While a practitioner's competence may be measured by examining different aspects of the patient-practitioner interaction, the way in which the practitioner touched the patient gave many of the participants a cue as to the technical skill of the practitioner.

For all of the participants an assessment was made about the technical competence of their practitioner. Furthermore, this assessment was essentially based on how the practitioner interacted with their bodies. For Phil this evaluation was conducted from the first moment that he made physical contact with his practitioner:

But certainly that first handshake in the way it is delivered automatically gave him a ... established his authority in a sort of calm way, which makes you relax. You get the feeling that this man knows what he is doing. The first moment I met him I thought, he knows what he is doing. I didn't sort of think about, you know, was he incompetent or something; it didn't cross my mind (Phil, p. 9).

From this comment it becomes clear that for Phil, the assessment of his practitioner's competence came before any physical assessment or treatment had occurred. While this evaluation most likely involved the assessment of other cues such as verbal and visual signs it also suggests the heavy weighting that Phil puts on the physical interaction which occurs. Furthermore, it could be hypothesised that this reliance on 
physical cues is generally reflective of the relationship that the participant and practitioner have.

In contrast, Sarah assessed her practitioner's competence based on the way in which techniques were conducted. In an attempt to explain how her practitioner's touch conveyed a sense of competence to her, Sarah compared her practitioner's touch with students who had given her massages before:

It's kind of an efficiency thing. But at the same time, when I was studying massage, we would always do swaps on each other and some people would move your arm up or down or on your leg or roll you over, and you could tell that they were quite confident and comfortable with it, were quite sure about what they were doing so therefore their touch conveyed that. You know their touch was firm but gentle but professional, placed in all the right places. You know, [they] didn't make you feel, oh my god we are going to lose the towel (Sarah, p. 8).

This reflection draws attention to the link between practitioner competence and professionalism. Sarah has an expectation that her practitioner will be competent and confident (see subtheme [C3] Confidence - very reassuring) as her physical therapist. Closely allied with this idea she believes that her practitioner should be comfortable with the touch they engage in. Thus there is close association between practitioner competence and safety (see subtheme [B2] Trust - a prerequisite for physical contact) within the therapeutic relationship.

As already mentioned a practitioner's competence was also assessed by almost all of the participants via their ability to identify and palpate areas of the participant's body that were significant for them. Phil said this of his practitioner:

He put his finger on spots and sort of found, and sort of worked around a bit but I think that seemed to be more identifying a focus or locus of whatever than a treatment thing. He seemed to have a knack for getting just in exactly the right spot (Phil, p. 7). 
It can be seen from this statement that Phil has a clear idea about what the practitioner is attempting to do. Furthermore it appears the practitioner's "knack" for finding the right areas built confidence in his practitioner's touch.

\section{[C3] Confidence - very reassuring}

Some of the participants identified confidence as a distinct feature of the practitioner's touch that reassured them of both the practitioner's competence and the therapeutic process. Like competence this feature of the interaction was conveyed to the participant through verbal, visual and physical cues and was closely linked with the professionalism of the practitioner.

Phil described this of his osteopathic practitioner when he greeted him at the start of each session:

He comes a long way to meet you to shake hand. [He] sort of fixes you with his eye and then sort of comes forward with his hand you know. Yes he sort of comes at you with his hand. Very sort of reassuring I think. Welcoming. He doesn't just shake your hand, he comes at you with a hand (Phil, p. 1).

Thus it can be seen from the above statement that it is the combination of the eye contact as well as the way in which the handshake is initiated which provides a sense of reassurance for Phil. For Sarah, it is the comfortableness of her practitioner's touch that engenders confidence in her practitioner. In addition, she makes a direct link between the importance of both confidence and professionalism and being comfortable in the therapeutic relationship:

And you know, I guess the whole, by being professional and confident and whatever and anything else you do, without being ... without making it uncomfortable (Sarah, p. 5). 
Phil also recognized his practitioner's confidence in the effortless way in which he handled his body. He identified his practitioner's confidence as a particularly reassuring feature of his technique when he described this about his practitioner's touch:

Reassuring I guess. And because his touch is so calm and confident, that transmits itself so that I stay calm about the impending manipulation (Phil, p. 5).

Thus it is clear from this statement that practitioner confidence not only reassures patients about the practitioner's skill but also aids the therapeutic process by allowing the patient to be able to fully relax.

\section{Summary}

The experience of touch for the participants in an osteopathic session is one of care and security. Theme [A] The Process - a physical interaction examined the importance of the physical interaction in communicating the practitioner's presence and support to the participant. Theme [B] Professionalism - a practitioner's responsibility conveyed the practitioner's personal and professional virtues while Theme [C] Reassurance $-a$ therapeutic necessity illustrated the ability of touch to communicate the practitioner's therapeutic competence and assurance. Overall, the participant's experience of touch was that it communicated both the practitioner's humanistic and professional attributes and skills.

The following chapter will discuss some of the ideas that arose from these themes and interpret the relevance of these findings in light of the current literature and thought. In the interests of structure, the following chapter will be divided into three sections to match each of the major themes from the current chapter. Each theme will then be divided into its subthemes for in-depth analysis and discussion. 
Chapter Six: Discussion 
The goal of this chapter is to discuss some of the themes and ideas that emerged from the data and to explore the relevance of these findings in light of the current literature. What this synthesis reveals is that touch mediates the practitioner-patient communication and enhances the therapeutic relationship. While many healthcare professionals use verbal communication to engage with, to support and to reassure their patients, the participants of the current study made it clear that they experienced these facets of the therapeutic interaction through the way in which the practitioner touched their bodies. Furthermore, the literature supports the idea that these elements of the therapeutic interaction enhance therapeutic outcomes and patient satisfaction.

Before continuing it should be acknowledged that the following discussion has a Western-centric view of healthcare and illness and thus discusses ideas that are relevant within the Western healthcare tradition.

\section{THEME [A]: The Process - a physical Interaction}

The analysis of this theme in the previous chapter highlighted the significance of the physical patient-practitioner interaction and its importance to the therapeutic process. Subtheme [A1] Engagement: let the session begin, symbolised the start of the session and indicated to the participants that the practitioner was beginning to engage with them and their body. Subtheme [A2] Dialogue: knowing hands converse with an expressive body acknowledged the reciprocal nature of the therapeutic interaction and the clear motives for the practitioner's touch. Finally, subtheme [A3] Support - being with the patient emphasized the importance of touch in communicating to the participant that the practitioner was completely available to assist the participant on their therapeutic journey. 


\section{[A1] Engagement - let the session begin}

As mentioned above, this subtheme highlighted the role of touch in indicating to the participants that the session had begun. Perhaps more importantly however, this first physical contact communicated to the participants that the practitioner was available and interested in connecting and attending to them and their body. This idea is consistent with the Concise Oxford English Dictionary ( $11^{\text {th }}$ ed., revised), which defines the verb engage as "attract or involve (someone's interest or attention)" (Soanes \& Stevenson, 2006, p. 472). Furthermore, the dictionary defines engagement as "an appointment" and "the action of engaging or being engaged" (p. 472). Thus the verb engagement is to attend to or be involved with something while the noun engagement is an appointment (in this theme it represents a professional appointment).

The idea of engaging with the patient at the start of a consultation is consistent across numerous clinical communication handbooks and manuals (Billings \& Stoeckle, 1999; Keller \& Carroll, 1994; Piasecki, 2003; Reifsteck, 1998; Silverman, Kurtz, \& Draper, 2005; D. Williams, 1997). For example the theme of engaging with the patient is consistent with the "E4 model" for clinical communication set out by Keller and Carroll (Keller \& Carroll, 1994). Their model describes specific behaviours and guidelines that the clinician can use to improve their clinical communication skills (Keller \& Carroll, 1994; Piasecki, 2003). Synthesised from an extensive review of the client-practitioner relationship the model consists of four "E"s - Engage, Empathize, Educate and Enlist. According to the authors these strategies are designed to improve the way in which clinicians communicate with their patients regardless of their discipline or experience.

Silverman et al (2005) have emphasized that the opening few minutes of a consultation are of critical importance to the clinical encounter. During this time the clinicians make their first impressions of the patient, establish rapport and attempt to identify the patient's problem (Silverman et al., 2005; D. Williams, 1997). At the same time the patient will make his or her own assessment of the practitioner's presentation. Consequently the beginning of the interview is a particularly important time for the clinician to communicate clearly and effectively (Keller \& Carroll, 1994; Piasecki, 2003; Silverman et al., 2005). Ironically however, research indicates that the initial 
phase of the interview is the time when breakdowns in communication are most likely to occur (Silverman et al., 2005).

Communication is therefore a key variable in the patient-practitioner relationship (Piasecki, 2003). Furthermore, communication in the literature has been conventionally classified into verbal and non-verbal behaviours. Non-verbal communication includes proximity, touch, eye-contact and eye-gaze, facial expression, gesture, body posture and head movements (D. Williams, 1997). Williams has described touch as the most important non-verbal behaviour for the health professional as it is often used to examine, diagnose and treat. Unsurprisingly, an observational study conducted by Roberts and Bucksey (2007) found that touch was the most common non-verbal behaviour used by physical therapists in their interaction with patients. It should be noted that in this study the researchers did not make a distinction between procedural and non-procedural touch and thus only limited conclusions can be drawn about the types of touch that therapists use.

Non-verbal behaviour also provides important information for the practitioner about the patient's demeanour and vice versa. Silverman et al. (2005) have noted that if a clinician does not pay attention to his or her own non-verbal cues, other verbal efforts to communicate may be undone. Furthermore, if verbal and non-verbal signals contradict each other, the practitioner risks confusing the patient and at worst the non-verbal message may win out (Roberts \& Bucksey, 2007; Silverman et al., 2005). This last point has particular relevance when revisiting the interviews as John (p.12) noted earlier that there is an inherent honesty about body language that makes it difficult to lie with your body.

The engagement theme also communicates to the participant that the practitioner is attending to them and is interested in the consultation. Silverman et al. (2005) say this about attention:

Although this may be just one of many routine consultations of the day for the doctor, for the patient it may be a far more important and significant occasion. The patient is usually entirely focused on the interview to come - it is clearly 
helpful if the doctor reciprocates with his or her full attention (Silverman et al., 2005, p. 38).

This is supported by Billings and Stoekle (1999) who point out that a patient will quickly notice at the start of an interview whether you seem "friendly, warm and personally interested in them" (p. 14). Furthermore both Silverman et al. (2005) and Billings and Stoeckle (1999) argue that there are a host of verbal and non-verbal clues that can indicate to the patient the practitioner's level of interest including the way in which they touch their patients.

The importance of the initial encounter and attending to the patient has also been identified in the physiotherapy literature. Gyllensten, Gard, Salford and Ekdahl (1999) conducted a qualitative study examining the clinical encounter from a physiotherapist's perspective. One of the major themes that emerged was an 'interaction' dimension. Within this theme different participants discussed the importance of establishing contact and co-operation with their patients, imparting a empathetic attitude, giving patients their full attention and treating them with respect, being sensitive and intuitive, listening, understanding the whole person and acknowledging body language and facial expressions. Moreover these skills were considered crucial to promoting patient responsibility and positive therapeutic outcomes. A more recent study by Gyllensten, Gard, Hansson and Eckdahl (2000) also highlighted the importance of being present in the moment and the use of non-verbal skills in the clinical encounter. ${ }^{21}$

Perhaps Silverman et al. (2005) best express the importance of the initial interaction when they say this:

We cannot emphasise enough the importance of taking steps to build the relationship from the beginning of the interview. Demonstrating interest, concern and respect for the patient and using appropriate non-verbal behaviour are so important in laying the groundwork for a productive and collaborative relationship (Silverman et al., 2005, p. 41).

\footnotetext{
${ }^{21}$ While the participants of this study were physiotherapists who worked in psychiatric care, it highlighted the universality of the importance of the practitioner's attentiveness and non-verbal skills in the clinical encounter.
} 


\section{[A2] Dialogue - knowing hands converse with an expressive body}

According to Ekerholt and Bergland (2006) during a physiotherapy session skin-to-skin contact can be described as "a kind of body dialogue, a dialogue without linguistic references" (p. 140). This concept is supported by the current subtheme, which explores the physical dialogue that occurs between the practitioner's hands and the participant's body. The data from the current study revealed that for the participants, touch not only communicated the practitioner's diagnostic and treatment goals but also communicated information about their bodies to the practitioner's hands and guided the treatment process. Thus this theme highlights the inherently bi-directional nature of touch.

As has been established communication is at the heart of a successful clinical interaction, and will often determine the success of the alliance formed between the patient and the therapist (Ekerholt \& Bergland, 2004; Keller \& Carroll, 1994; Piasecki, 2003; Stewart, 1995). The significance of this idea to the current theme is that there is something intrinsic to the touching process whereby the meaning of the practitioner's touch is naturally conveyed to the participants. In addition this is often supported by the practitioner's verbal cues. The result of this is that the participant feels informed about their treatment, which can bring a greater sense of control for the patient. This is important because there is often a power discrepancy in clinical interactions that can make the patient feel vulnerable. Thus touch facilitates a more mutual or reciprocal interaction. Additionally, the participant's understanding of the practitioner's touch also reinforces the theme discussed above that the practitioner is engaged with the participant and is attending to their body.

The other feature of this physical dialogue articulated by some of the participants was the mutual or bi-directional nature of it, whereby they were conscious of the diagnostic information that their body was actively giving the practitioner. This feature of the communication is in stark contrast to many clinical interactions that occur in other healthcare settings. Purtilo and Haddad (2007) have noted that "most interactions with patients take the form of an "interview" rather than a conversation or dialogue" (p.163). They argue that the reason this occurs is that health professionals have been taught from very early in their medical training the importance of the "interview" technique for 
gathering information. Williams (1997) notes that what distinguishes these "interviews" from a conversation is that the flow of information is in one direction and is controlled by the interviewer (i.e. the doctor). However, more recent healthcare models such as the patient-centred ${ }^{22}$ and biopsychosocial approaches actively involve the patients in the treatment and rehabilitation process and thus require a bi-directional interaction (Gyllensten et al., 1999; Mead \& Bower, 2000; Mead, Bower, \& Hann, 2002; Ong et al., 1995).

The patient-centred concept is seen as an approach to healthcare that "emphasises on the part of the health professionals, attention to patients' psychosocial (as well as physical) needs, the use of psychotherapeutic behaviours to convey a sense of partnership and positive regard, and active facilitation of patients' involvement in decision-making" (p. 283, Mead et al., 2002). What this data suggests is that through touch the practitioner utilises a "psychotherapeutic behaviour" which conveys a sense of "partnership" to the participants, whereby the participant feels actively involved in the diagnostic and treatment processes. Thus it appears that touch's inherent bi-directional quality facilitates and encourages a patient-centred approach to healthcare.

The idea of a dialogue between the practitioner's hands and the participant's body was also present it the study discussed above (in the literature review) by Ekerholt and Bergland (2006) which examined the patient's experience of massage in Psychomotor Physiotherapy. In this study the authors also reported a non-verbal dialogue between the practitioner and their patients. In addition, the authors explained that for the participants (expert physiotherapists) this physical dialogue was supported by verbal communication, which in combination improved the therapeutic process. Similarly, participants in the current study supported the idea that the combination of the physical and verbal communication enhanced the therapeutic experience.

Another feature of this theme that the data illuminated was the responsive nature of the practitioner's touch. The participants explained that rather than just a routine

\footnotetext{
${ }^{22}$ According to Mead and Bower (2002), the literature has lacked consensus on the meaning of a patientcentred approach. While a full discussion of this concept is outside the scope of this paper Mead and Bower have suggested that the patient-centred approach consists of five dimensions - the biopsychosocial perspective, the patient as person, sharing power and responsibility, the therapeutic alliance and the doctor as person.
} 
examination and treatment they felt their practitioners listened to their bodies and adjusted their treatments in reaction to their particular structure. Similar to the engagement theme above, this listening and adjusting quality of the practitioners' touch instilled a sense of the practitioner engaging with them.

This idea of listening to the patient's body and adapting the treatment in response to the patient is consistent across physical therapy literature (Hartman, 1997; Parsons \& Marcer, 2006). Osteopathic textbooks highlight the importance of treating the individual by listening with the fingers and adjusting techniques to the requirements of the tissues. However, this listening quality is not unique to osteopathy as other physical therapy literature also refers to this skill (Gyllensten et al., 1999; Roger et al., 2002). For example Gyllensten et al. (1999) conducted a qualitative study that examined the physiotherapist's perspective on the clinical encounter. One of their findings was that physiotherapists consider it important to be sensitive and intuitive to the patient's body and to adapt techniques when appropriate.

According to Crellin (2005), patients have a real need to feel listened to. He says "[1]istening that hears and identifies concerns and worries, perhaps from intonations, may well help with 'healing' by finding meanings to an illness, connections for the patient and showing empathy". While Crellin is probably referring to the importance of auditory listening, it could be suggested that given the tactile nature of the communication which occurs in the osteopathic interaction, palpatory listening may achieve a similar result for the patient.

\section{[A3] Support - just holding}

This subtheme relates to the way in which touch reinforces the participant's sense of support and its importance to the therapeutic process. As mentioned in the analysis, this support manifested for the participants in different ways. While some of the participants described their practitioner's supporting approach, other participants identified specific techniques that engendered in them a sense of being supported or 
reassured. It was also noted that this support was experienced on both a physical and psychological level and translated into a feeling of being cared for.

As discussed above, Harlow's (1958) early research with infant monkeys demonstrated the importance of maternal contact for the healthy growth and development of an individual. Subsequently, Bowlby (1988) developed an attachment theory which described the quality of parent-infant physical contact as central to the development of a secure, care-giving environment. As the infant grows it is also possible to see the importance of physical contact in providing support and comfort. For example a child who stubs their toe or grazes their knee is often rewarded with a comforting hug from their mother or father. While this may serve to distract the child, it also supports the child and reassures them that the parent is close by.

The idea of touch providing support however is not just relegated to the confines of early life (Chang, 2001). According to Montagu (1986) tactile stimulation appears to be a fundamental requirement for the healthy development of an individual throughout the lifespan. Furthermore fulfilling this "need" in individuals, both young and old, may encourage the conviction that they are valued, wanted, supported and connected to a network of others.

While it appears optimistic, Barritt (2005) has stated that a healthcare practitioner's ability to facilitate healing is built on the premise that they understand the human reaction to illness. Susan Sontag (1977) writes:

Illness is the night-side of life, a more onerous citizenship. Everyone who is born holds dual citizenship, in the kingdom of the well and in the kingdom of the sick. Although we all prefer to use only the good passport, sooner or later each of us is obliged, at least for a spell, to identify ourselves as citizens of that other place (Sontag, 1977, p. 3).

This quote from Susan Sontag's (1977) Illness as Metaphor beautifully captures the struggle that individuals bear when facing illness. Other professionals compare illness to the experience of bereavement or loss - loss of confidence, loss of control, loss of 
innocence and loss of omnipotence (Barritt, 2005). Thus intuitively it could be said that one of the key roles of health practitioners is to support and reassure patients in times of illness or loss. This idea is supported by Montagu (1986) who remarks that "[t]aking almost anyone's hand under conditions of stress is likely to exert a soothing effect, and by reducing anxiety give both the taken and the taker a feeling of greater security" (p. 226).

The idea of practitioners supporting their patients is also consistent with a general move away from the paternalistic attitude that once pervaded the medical model (Mechanic, 1998; Ong et al., 1995). Patients are now more informed and active in their own healthcare and the practitioner-patient relationship is more about support and facilitating the patient back to health. Interestingly one of the core tenets that has governed the osteopathic profession since its inception in the late nineteenth century is that the body has its own self-healing mechanisms (Parsons \& Marcer, 2006; Seffinger et al., 2003). Furthermore, it is the primary role of the osteopathic practitioner to facilitate health within the individual rather than to work toward trying to "heal" the patient.

The role of practitioners providing support for their patients has also been a consistent theme in physiotherapy practice literature (Kumlin \& Kroksmark, 1992; Roger et al., 2002). Kumlin and Kroksmark's study investigating a patient's first encounter with a physiotherapist reported this about the physiotherapist's intentions:

Each physiotherapist strives to create a safe and secure situation for the patient so that the patient will have confidence in her. This confidence creates the basis for the patient's involvement and concern in his own treatment and thereby activating his own internal resources (Kumlin \& Kroksmark, p. 41).

This idea of support was reinforced by the physiotherapists in Roger et al.'s (2002) study (see the current study's literature review) which reported using touch for the purposes of conveying security or safety. The authors defined security touch as "touch used to provide a feeling of safety or reassurance to patients, regardless of whether or not it was physically needed" (p.180). Furthermore, the physiotherapists reported that this form of touch was used most with patients who presented as anxious or nervous. 
In the current study, the support theme also alluded to the idea of the practitioner's touch being in some way accepting of the participant's body and the sense of support that this engendered. This finding is consistent with the study by Ekerholt and Bergland (2004) who investigated patient's experiences of Norwegian psychomotor psychotherapy. According to the authors their study indicated that an important element in a successful therapeutic session was the patient's feeling of being acknowledged, believed, understood and accepted by their therapist.

Another feature of the support theme was the idea of presence (having the practitioner physically with them) and the support that this provided. While there is a sparse amount of research in the physical therapies literature on the subject of presence, one field of healthcare that has thoroughly examined this idea is nursing. FinfgeldConnett's (2006) meta-analysis of the nursing literature on presence explains that it is a complex process that can occur in moments or over days, weeks and years. She suggests that presence involves being with another person in an intimate way. It normally involves close physical contact and includes "engaged availability, affectionate touching an attending to personal needs" (p.710). Furthermore, she explains, "this process is characterized by interpersonal sensitivity and is holistically focused on each individual's physical, psychological and spiritual well-being" (p. 710). The review also supported the idea that patients who experience presence report decreased stress and enhanced mental well-being as well as increased feelings of safety, security and improved self-esteem.

This section has examined the role of touch in mediating the physical relationship that exists between the practitioner and the patient and its importance to the therapeutic interaction. The next section adds to this analysis by thoroughly exploring the way in which the practitioner's touch facilitates a professional interaction that encourages a caring and trusting relationship between the practitioner and the participant. 


\section{THEME [B]: Professionalism - a practitioner's responsibility}

The analysis of this theme in the previous chapter revealed the importance of the professional interaction for the participants and highlighted patient expectations surrounding the physical contact that occurs in an osteopathic session. In this section Subtheme [B1] Care - an empathic quality will examine how touch is able to communicate care and how this is a constant feature across the healthcare literature. Subtheme [B2] Trust - a prerequisite for physical contact explores the importance of trust in building a strong healthcare relationship while subtheme [B3] Boundaries - $a$ professional relationship discusses the importance of boundaries in the healthcare setting and how touch can be used to establish safe clinical boundaries.

\section{[B1] Care - an empathic quality}

This subtheme relates to the way in which the practitioner's touch engendered in the participants a sense of being cared for within the participants. In the current study care was expressed through an understanding of the participant's physical narrative (that is through an understanding of where their body had been) while others articulated a degree of tenderness in their practitioner's touch which conveyed a sense of care. For many of the participants this sense of care was directly related to an empathic quality in the participant's touch. It was also clear from the interviews that this sense of care was mediated by both verbal and non-verbal communication.

In a sense, touch is the very first means by which caring is communicated between two people. Through early maternal contact an infant is constantly provided with love and nutrition and protected from danger (Montagu, 1986). And according to Nathan (1999), for an infant, the revelation of being loved, valued, safe and belonging are all mediated by the early physical contact which they receive. Moreover, Nathan states that these early memories are "stored in the adult as a vast data-bank of emotio-tactile and kinaesthetic sensations, instincts and reactions" (p.96) that can resurface during adult- 
to-adult touching when there is a significant discrepancy in power such as when a stranger is caring for you.

While this idea about early tactile experiences is relatively recent, the idea of caring as a central feature within healthcare is, in a sense, centuries old. The Hippocratic oath for example, traditionally taken by every physician entering the medical field, states that "I will use my power to help the sick to the best of my ability and judgement; I will abstain from harming or wronging any man by it" (cited in Porter, 1997, p. 63). Although no longer taken by all healthcare professionals, modern ethical codes of conduct, which guide practitioners such as osteopaths, include a similar provision guiding healthcare practices. The Code of Ethics as stipulated by the Osteopathic Council of New Zealand (2006) states that the osteopath must "make the care of the patient their main concern" and "understand the concept of duty of care and associated responsibilities". For the current study however an exploration of the theme of care reveals that for the participants, touch communicates practitioner care, whereby they understand that the practitioner is personally looking after them.

According to Nathan (1999) the idea of human beings using touch to comfort each other in times of physical or emotional distress has been around "since time began" (p.94). Human beings touch, hold and hug people when in distress, despair or in some other way vulnerable. Nathan describes this as "an attempt to lower the level of another's pain by sharing embodiment" (p. 94). It is not surprising then that the touch received from a healthcare professional is experienced by many as inherently caring.

Research on healthcare professionals caring for their patients is perhaps best attested within the nursing literature. According to Kyle (1995) caring is viewed in the literature as a central feature of nursing practice. However, review authors have also consistently identified the difficulties that nursing researchers have had in defining the concept of caring (Finfgeld-Connett, 2008; Kyle, 1995). In an attempt to demystify this concept Finfgeld-Connett (2008) conducted a meta-synthesis of the nursing literature and found that caring within nursing was an "interpersonal process that is characterised by expert nursing, interpersonal sensitivity and intimate relationships" (p. 3). She also cites 
literature that shows interpersonal sensitivity is a process of being physically and mindfully present, centring on the patient and being intuitive and empathetic.

While there is a scarcity of research into caring in physiotherapy literature caring has been identified as a key area of physical therapy practice (Greenfield, 2006). Jensen, Gwyer, Shepard \& Hack (2000) conducted a qualitative grounded theory study to identify the dimensions of clinical expertise in physical therapy practice. From the study the authors developed a theoretical model of expert practice within which one aspect was "consistent virtues seen in caring and commitment to patients". To support this the authors noted this about the expert clinicians who participated in the study “consistent with their interactions with patients was the experts' ability to communicate a sense of commitment and caring about the patient. These therapists saw patient advocacy as a vital professional role..." (p. 39).

The physical therapy literature has also contributed understanding to the role of touch in communicating care. The physiotherapist participants in Roger et al.'s (2002) study into physiotherapist's use of touch frequently reported using a caring touch to convey comfort, emotion or support. Assistive touch was used most frequently. In contrast, participants in Helm, Kinfu, Kline and Zappile's (1997) study investigating the same phenomena reported caring touch as the most frequently used. It is interesting to note that the qualitative methodology (interviews) utilised in the latter study lead to a greater percentage of reported caring touch compared to the former study that used a quantitative approach (video-analysis). One explanation is that physical therapists believe they use more caring touch than they actually do but it may also relate to the difference in methodology. Use of an interview does not encourage the participant to distinguish between verbal and non-verbal communication, while a video analysis forces the participants to distinguish care that is communicated through purely physical communication. Thus the difference in these two studies may reinforce the idea that care is often a complex interplay between verbal and non-verbal communication.

When comparing the findings of the current research with studies outlined above it is important to remember a number of differences in study design. Firstly, the current study was a qualitative exploration of the patient's view of touch rather than an 
investigation of the practitioner's viewpoint. This is significant as the way that care is perceived may be vastly different from how it is bestowed. Secondly, while a video analysis in Helm et al's (1997) study may have revealed touch that the participant was able to specifically label as caring touch, the qualitative nature of the current study enabled participants to describe the experience of this care and how touch contributed to this feeling.

\section{[B2] Trust - a prerequisite for physical contact}

This subtheme explored how the practitioner's touch contributed to building a trusting therapeutic relationship between the participants and their practitioners. According to the Concise Oxford English Dictionary ( $11^{\text {th }}$ ed., revised) trust means a "firm belief in someone or something" (Soanes \& Stevenson, 2006, p. 1549). This is consistent with the findings of the current study which found that the participants had a strong belief in their practitioner and indeed trust was considered a prerequisite for seeking their practitioner's consultation. As with subtheme [B1] care - an empathic quality, trust appeared to be a consequence of both the physical and verbal dialogue as well as contributing to good therapeutic outcomes.

Trust is recognised as an important precursor for a successful therapeutic relationship (Sim, 1986; Thom, 2001; Thom \& Campbell, 1997) and in fact has been identified as a key component of professionalism (Barritt, 2005; Makely, 2005). However, it has also been noted that the idea of trust in the literature is often an ambiguous scientific concept which lacks a consistent definition (Hupcey, Penrod, Morse, \& Mitcham, 2001). Within the healthcare literature a number of different definitions have been proposed (Hupcey \& Miller, 2006). Johns (1996) describes trust as a "[w]illingness to place oneself in a relationship that establishes or increases vulnerability with reliance upon someone or something to perform as expected" (p. 81) while Hupcey and Miller (2006) defined trust as emerging:

... from the identification of a need that cannot be met without the assistance of 
another and some assessment of the risk involved in relying on the other to meet this need. Trust is the willing dependence on another's actions, but is limited to the area of need and is subject to overt and covert testing. The outcome of trust is an evaluation of the congruence between expectations of the trusted person and actions (Hupcey \& Miller, 2006, p. 1135).

Despite slight differences in these two definitions, what appears consistent is that the process of trust requires an individual to make himself or herself vulnerable by placing themselves in the hands of another person.

In an attempt to understand how trust affects the patient-practitioner relationship, Thom and Campbell (1997) and Thom (2001) conducted research examining how physician's behaviour affected trust. In an initial exploratory study using focus groups Thom and Campbell (1997) reported that trust was a consequence of the physician's professional competence as well as their interpersonal skills. These findings were subsequently supported by a quantitative study (Thom, 2001). This study reported that behaviours which increased trust in a physician included being comforting and caring, demonstrating competency, encouraging and answering questions, and explaining. Significantly, Thom reported that caring and comfort were as important as technical competency for their participants in generating trust.

Nurse researchers have also explored the concept of trust within the therapeutic relationship (Hupcey \& Miller, 2006; Hupcey et al., 2001). Trojan and Yonge (1993) used grounded theory to identify four phases that occurred in the development of trust between nurses and elderly clients - initial trusting, connecting, negotiating and helping. Consistent with the findings from Thom and Campbell (1997) reported above, behaviours identified as important to the development of trust included both interpersonal skills as well as technical competence. A more recent qualitative study conducted by Hupcey and Miller (2006) supported these findings with an investigation of community dwelling adults' trust in local healthcare providers. The researchers reported that while trust was developed through having confidence in the provider's competence, it was also strongly connected with the interpersonal caring attributes of the provider. 
Despite the importance of trust to the physical and manipulative therapies, a search of the literature reveals a scarcity of research specifically examining the role of trust or its development within the patient-practitioner relationship. However there was one phenomenological study conducted by Lee-Treweek (2002) which examined trust within complementary therapies. In this study Lee-Treweek interviewed sixteen patients of cranial osteopathy, asking them about the development of trust with their practitioner. In contrast to the research reported above, Lee-Treweek argued that it is not the practitioner and their therapy that is the basis of trust within complementary therapies but a combination of network trust (trust relying on the accounts of other people) and the phenomenological work of the patient following the treatment as they strive to seek meaning in the treatments they experience. Thus for these participants the development of trust involved a reflective process after the session.

While the literature cited above examines how trust is generated, it is still unclear to what extent touch contributes to the development of trust within an osteopathic session. It is worth remembering that the patient-practitioner relationship is built on verbal and non-verbal communication and that if verbal and non-verbal cues are contradictory then the non-verbal message will predominate (Frank, 1957; Silverman et al., 2005). Considering this, it could be suggested that there is something inherently truthful about touch as a form of communication. This is supported by Nathan (1999) who comments that "It [touch] usually communicates directly an attitude which expresses the toucher's (often subconscious) genuine sentiments" (p.89). Thus for a patient, the very nature of touch and its communicative function within the session is likely to engender a sense of trust in their practitioner.

Finally, it is worth briefly discussing the relationship between touch and power in the therapeutic relationship. Power is often present in the therapeutic relationship because a vulnerable individual is reliant on a stranger's skill and expertise (Nathan, 1999). With touch however, the gulf that is created by the power discrepancy can be bridged and in response the patient's vulnerability, anxiety and fear are diminished. According to Gadow (cited in Nathan, 1999) this can be achieved because the touch exposes the toucher's own vulnerability and thus a mutuality of trust and experience is created. 


\section{[B3] Boundaries - a professional relationship}

This subtheme relates to the way in which touch mediates some of the professional boundaries that exist in the patient-practitioner relationship. The participants in the current study had clear ideas about the appropriateness of touch in the physical relationship and were also conscious of the ability of touch to develop emotional connections that were inappropriate for the patient-practitioner relationship.

The success of any therapeutic interaction is dependent on the relationship that exists between the patient and the practitioner. It may also be said that the success of any therapeutic relationship is similarly dependent on the establishment of robust and transparent boundaries (Pye \& Jago, 1998). Thus boundaries form the basis of professionalism and allow both individuals in the therapeutic interaction to concentrate their respective energies on the therapeutic process.

The skin forms a natural shell around an individual and more importantly a boundary between an individual and their environment (Leder, 1992). In other words, the skin forms a boundary of the self. It is also important to understand an individual as both having and being a body (Ekerholt \& Bergland, 2004, 2006) and that to a touch a person's body is to touch their being (Nathan, 1999). Bertrand Russell once wrote "[n]ot only our geometry and our physics, but our whole conception of what exists outside us is based upon the sense of touch" (Russell, 1977, p. 10)). Reflecting on this it becomes clear that medical professionals must be respectful of the patient's physical boundary and the significance of touching it.

According to Bowers (2000), intimacy appropriately expressed in context and content is essential to the practitioner-patient interaction. However she also warns that " $[\mathrm{t}]$ ouching is the intimate behaviour of doctors most charged with meaning, most open to misinterpretation, and most likely to lead to transgression" (p. 15). Furthermore, Bowers emphasises that it is the practitioner's responsibility to ensure that patients understand touch as it relates to the diagnostic or therapeutic process. 
Research in the physical therapies has also supported the supposition that touch plays a role in establishing distance in the clinical relationship. One theme that emerged in Ekerholt and Bergland's (2004) study (discussed in the literature review) is the importance of personal boundaries. The authors suggested that given the vulnerability of the patients in a physiotherapy session, practitioners should be sensitive to the patient and create a safe environment for the establishment of secure foundations for the therapeutic relationship.

The question then arises why is touch a potential threat to this boundary of the self? According to Nathan (1999) physical closeness or touching is existentially challenging. As alluded to above, the sense of what is self, is initially defined by the realisation what is self and what is non-self. This naturally leads to the individual's understanding of boundaries between themselves and others. Nathan (1999) also suggests that physical proximity or touch with a relative stranger, such as a physical practitioner, has the potential to threaten an individual's sense of self.

Nathan (1999) has tried to explain this threat to the self by highlighting the fact that touch is the only sense that is completely unmediated - it is direct and tangible. Because of this, the act of touching someone is inherently intimate. In fact Nathan suggests that "leaving aside for a moment the assertive and aggressive connotations of touching, as a rule, it symbolises/expresses degrees of, and also is intimacy" (p.91).

A discussion of this theme has drawn attention to the significance of touch in establishing and defining the professional relationship. While it is important for the practitioner to communicate care and trust through touch, it is also critical for them to convey a strong sense of boundaries through the way in which they interact with the patient's body. The next theme reinforces the goals of the therapeutic interaction by exploring the importance of touch in communicating a sense of reassurance to the participant. 


\title{
THEME [C]: Reassurance - a therapeutic necessity
}

\author{
The Mask \\ He lies still \\ trapped on the table \\ head covered \\ in clear plastic \\ flexible not malleable \\ unable to move \\ he waits \\ beneath the mask who knows \\ apprehension, excitement \\ acceptance \\ a thief \\ a husband \\ a child molester \\ a Samaritan \\ the mask offers its \\ succour to all \\ the plastic is littered \\ with archaic symbols \\ like a Masonic star or \\ a fleur de lys \\ membership of the club \\ restricted to \\ those who can make \\ meaning of the signs. \\ the white coated voices \\ return, hands on \\ soft hands \\ gentle hands \\ talking hands \\ hands that bring \\ the comfort of competence.
}

Mike Consedine (May 2007)

This poem was written by my father following his experiences in the radiotherapy ward and has been included here because it highlights a number of features that are relevant 
to theme [C] Reassurance - a therapeutic necessity. For the participants this theme highlighted the role of touch in reassuring them about both the practitioner's theoretical knowledge as well as their technical skill. Furthermore the communication of reassurance spanned diagnostic, interpretive and treatment touch and gave the practitioner a professional credibility.

This discussion illuminates the important role of reassurance in healthcare and how knowledge, competence and confidence are conveyed by different healthcare professionals. Subtheme [C1] Knowledge - with seeing fingers looks at the importance of palpation to osteopathic practice. Subtheme [C2] Competence - a knowing touch examines the significance of presumed practitioner knowledge while subtheme [C3] Confidence - very reassuring examines the importance of remaining confident for vulnerable patients.

\section{[C1] Knowledge - with seeing fingers}

As mentioned above this subtheme relates to the way in which the participants could detect their practitioner's anatomical knowledge and intention with particular techniques. The Concise Oxford English Dictionary (11 ${ }^{\text {th }}$ ed., revised) defines knowledge as "information and skills acquired through experience or education" and an "awareness or familiarity that is gained with experience" (Soanes \& Stevenson, 2006, p. 789). For many of the participants the two elements of experience and education were present in their practitioner's touch and manifested as an informed and responsive touch that was distinctive of osteopathy. Moreover, these elements of the touch offered some reassurance to the participant about the competence of their practitioner.

The current research supports the idea that palpation is an important part of osteopathic diagnosis and treatment. Sutherland (cited in Comeaux, 2005) once described an osteopathic practitioner's correctly and lightly placed hand as "feeling, seeing, thinking and knowing" (p.151). Research has also suggested that physical therapists view palpation as a unique form of touch whereby information can be both gathered and 
communicated (Barnes, 2004; Roger et al., 2002). For example Roger et al.'s (2002) study examining a physiotherapist's use of touch revealed a theme of perceiving information which was described as touch that was used to gain information about patients' diagnoses or symptoms. In addition the participants in Helm, Kinfu, Kline and Zappile's (1997) study discussed above, also reported using a task-oriented touch "to perceive information or to provide information to the patient through physical contact" (p.20).

Theme [C1] Knowledge - with seeing fingers also suggests the art of careful palpation provides some therapeutic benefit by reassuring the patient of their practitioner's skill. According to Nathan (1999), "where touch is used mainly expressively, it expresses feelings, attitudes and sentiments. Where it is used mainly as procedure or technique, it demonstrates knowledge, thought and belief' (p.91). This idea is also supported by Cooper (1996) who says this about the therapeutic examination in medical practice, "while it is true that some conditions can be assessed without a physical examination (angina is a good example), patients do not know this, and a careful physical examination, focusing on the part complained of, demonstrates that you have gained an understanding of the problem" (p. 695). Thus for the patient, the ability to see and feel the physical examination process contributes to a feeling that the practitioner is skilful and knowledgeable and understands their particular condition.

\section{[C2] Competence - a knowing touch}

This subtheme relates to the way in which the practitioner's touch inspired confidence in the participants about the technical skills of their practitioner. The Concise Oxford English Dictionary $\left(11^{\text {th }}\right.$ ed., revised) defines competent as "having the necessary ability or knowledge to do something successfully; acceptable and satisfactory" (Soanes \& Stevenson, 2006, p. 292). This definition is consistent with the participants' experience, that their practitioner's touch conveyed a sense of both technical ability and anatomical knowledge (see subtheme [C1] Knowledge - with seeing fingers). Moreover, this idea of the practitioner's competence spanned the entire session, from the initial handshake 
to the way in which the practitioner located painful areas and performed different treatment techniques.

Being a competent practitioner, with adequate technical knowledge and skill, is clearly a prerequisite to practice in any healthcare profession (Bendapudi et al., 2006). Competence in a medical sense consists of knowledge and technical ability but also includes interpersonal skills such as making a patient feel at ease and sensitive interviewing skills (Mechanic, 1998; Rethans, Sturmans, Drop, Van Der Vleuten, \& Hobus, 1991).

According to Barritt (2005) physicians are now required to earn public respect. Given that patients have a choice about the healthcare practitioner they consult, it is natural for them to assess the competence of their practitioner. In fact literature suggests that the nature of medical services turns patients into detectives who are looking for clues about the practitioner's competence and care (Mechanic, 1998; Potter et al., 2003). However, in an effort to improve healthcare delivery researchers have also begun to examine what qualities a patient looks for in their physician to provide quality healthcare (Fung et al., 2005). This assessment involves an evaluation of both the practitioner's technical and interpersonal skills.

To date there are still discrepancies in the literature related to the relative importance of a physician's technical versus interpersonal skills. For example Fung et al. (2005) found that when participants were presented with a hypothetical physician they showed a strong preference for physicians with high technical competency when forced to make a trade-off between the two skills. In contrast, Bendapudi et al. (2006) conducted a qualitative investigation of patient's perspectives on ideal physician behaviours and found that interpersonal skills such as being confident, empathetic, humane, personal, forthright, respectful and thorough, rated more highly in their interviews than technical skills. What is interesting is that the interpersonal skills identified by the participants in Bendapudi et al.'s study correspond to some of the behaviours that are conveyed by touch in the current study. This may suggest that touch works on two levels to convey competence, firstly at a technical level through the practitioner's skill and secondly at a non-verbal communicative level. 
The theme of professional competence has also been explored in the physical therapies literature (Ekerholt \& Bergland, 2004; Gyllensten et al., 1999; May, 2001). A theme in Ekerholt and Bergland's (2004) study, discovering professional skills, stressed the importance for the participants of being sure of their practitioner's skill and competence in his or her field of work. The authors said this of the participants "[t]he informants pointed out that the personality of the therapist is not enough: the therapist must also radiate professional security and competence" (p. 406). The physiotherapists in Gyllensten's (1999) study expressed their competence in a slightly different way. According to the authors the participants did not single out the therapeutic techniques as important in the interaction with the client but rather believed techniques to be background prerequisites that must be fully integrated so that the physiotherapist can feel free to respond intuitively to the patient as a whole.

When asked to recall their initial visit with a cranial osteopath participants in LeeTreweek's (2002) study also cited the practitioner's handling of their body as an important element of their sense of his competence. The author also found a strong link between the patient's trust in their practitioner and the perceived competence and confidence.

Finally, this theme also suggested that the practitioner's ability to locate the symptomatic area(s) also added to the participant's sense of the practitioner's competence. This is consistent with Lee-Treweek's study, which reported that participant's confidence in the practitioner appeared to be related to the way in which he could locate painful areas without their prompting or feedback. This ability of the practitioner may have had an additional therapeutic value of validating the patient's complaint. Ekerholt and Bergland's (2004) have suggested that it is vital for patients to feel believed, understood and confirmed with regard to both their physical and emotional symptoms and complaints. 


\section{[C3] Confidence - very reassuring}

The subtheme [C3] Confidence - very reassuring explored the idea that the practitioner's touch conveyed to the participants a sense of confidence that reassured them of their practitioner's professionalism and competence. In addition, it was noted that there was a close association between a practitioner's confidence and the trust that participants felt toward their practitioner.

Reassurance is an important part of medical practice (Cooper, 1996; Laakso \& Niemi, 2004). In fact the Canadian physician William Osler (1849-1919) once said "Taking a lady's hand gives her confidence in her physician" (p. 226, cited in Montagu, 1986). Reassurance is important because when an individual presents to a healthcare professional it is likely that they are in pain or distress and are experiencing a high level of anxiety about their condition. A confident practitioner however, can reassure the patient by communicating a sense of control and security to the patient (Bendapudi et al., 2006; Hupcey et al., 2001; Laakso \& Niemi, 2004). Some literature also suggests that practitioner confidence has become more important recently because of the decreasing confidence patients have in the healthcare system (Mechanic, 1998).

It also makes sense that patients want to see their practitioner confident in roles or skills that are connected with their field of expertise. Thus for patients seeing a physical therapist, they expect to see physical examination and treatment techniques carried out competently and confidently.

As mentioned earlier the practitioner-patient interaction and communication has been well researched in the medical literature (Barrier et al., 2003; Bendapudi et al., 2006; Ong et al., 1995). Within some of this research it is possible to perceive a clear theme of the importance of practitioner confidence. Bendapudi et al.'s (2006) study identified practitioner confidence as one of seven behaviours that was sought and valued by their participants. In addition the authors found that a physician's assured manner engendered trust in the therapeutic relationship and a sense of relaxation within the participants. These themes are consistent with Phil (from the current study) who 
reported that because of his practitioner's calm and confident manner he was able to remain calm about any impending manipulations.

Other authors have also made the logical link between a practitioner's perceived confidence and their patient's trust (Hupcey \& Miller, 2006; Hupcey et al., 2001). However, Hupcey et al. have pointed out that while confidence is a concept closely allied to trust, it does not necessarily involve placing oneself in a dependent position or covertly or overtly testing the trustworthiness of the practitioner (both of which are involved with trust).

Another reason that confidence may be a useful practitioner behaviour relates to the placebo effect. The current clinical thinking about the placebo is that it does not simply refer to an inert intervention but also to a complex interplay of psychological factors in the patient-practitioner relationship (Barrett et al., 2006; Donnelly, 2004). The placebo effect "is taken to mean not only the narrow effect of a dummy intervention but also the broad array of nonspecific effects in the patient-physician relationship, including attention; compassionate care; and the modulation of expectations, anxiety, and selfawareness" (p. 817, Kaptchuk, 2002). Thus the practitioner's presence is a powerful placebo that can effect patient perception and confidence. In support of this a study conducted by Thomas (1987) found that patients who were seen by a positive practitioner (that is a practitioner who was optimistic and gave a confident diagnosis) recovered faster than those individuals seen by a negative practitioner.

Confidence has also been identified in the physical therapy literature as an important feature in creating a safe patient-practitioner interaction (Kumlin \& Kroksmark, 1992; May, 2001; Potter et al., 2003; Roberts \& Bucksey, 2007). The physiotherapy participants in Kumlin and Kroksmarks' (1992) study for example reported wanting to create a safe and secure environment in their first session with a patient to stimulate the patient's confidence in them. Furthermore, the physiotherapists used this patient confidence as a springboard for engaging the patients in their own therapy and activating their internal resources. In addition, Roberts and Bucksey (2007) noted in their observational study that inexperienced physiotherapists used fewer affective behaviours to engage with the patient because "therapists with less experience often 
lack confidence in their clinical abilities and so tend to focus on treatment techniques rather than more affective components, such as patient feelings" (Roberts and Bucksey, 2007, p. 592).

One of the themes in Lee-Treweek's (2002) study: trust in the treatment room also identified confidence as an important quality for the practitioner. Consistent with the current study, Lee-Treweek reported that participants appeared to gain confidence from the perception that the practitioner worked with the body in a capable way. Specifically, they highlighted the ability of the practitioner to locate painful areas with little input from the patient. The participants also alluded to the relaxing quality of this confidence in their practitioner.

The importance of confidence in the medical practitioner is perhaps best summarised by Barritt (2005) who states that "a certain level of confidence is required to motivate the healthcare professional to turn out of bed every morning, noon and night" (p. 190). He goes on to explain that healthcare professionals constantly live in a world of uncertainty and will inevitably make mistakes when treating patients. He stresses however the importance of remaining humbled rather than disheartened by this state of affairs.

A discussion of this theme has shown how the practitioner's medical and anatomical knowledge, competence and confidence are qualities of touch that are highly sought after by patients. Furthermore, the literature suggests that these qualities of touch significantly contribute to reassuring the patient of the practitioner's skill and their own condition.

Overall this discussion has examined the information and practitioner qualities that are conveyed to the patient through the medium of touch. As has been discussed in the analysis section, the participant's experience of touch was that it communicated the practitioner's humanism and professionalism. In his book 'Humanity in Healthcare the heart and soul of medicine' Barritt (2005) has listed seven virtues of being a healthcare professional - truthfulness, trustworthiness, insight, respect, vulnerability, compassion and enthusiasm. Interestingly, it appears from this research that touch 
forms a large part of the communication that is able to convey all of these qualities from the practitioner to the patient in an osteopathic session.

\section{Summary}

Chapter six has examined the implications of the findings of the current study in light of contemporary research and thought. It has been revealed that communication is at the heart of a successful clinical interaction and therefore has a large impact on the success of the therapeutic relationship formed between the practitioner and the patient. Furthermore, research has suggested that touch is the most important non-verbal behaviour for health professionals as it is often used to examine, diagnose and treat patients. However, this discussion has also offered additional explanations as to why the communicative functions of touch are important during an osteopathic session. Firstly, by its very nature, touch involves the patient in their own treatment in a way that makes them feel accepted and listened to. Moreover, the unmediated nature of touch endows an inherent honesty to the information conveyed through this form of nonverbal behaviour. Secondly, touch has an ability to connect the patient and the practitioner. This quality engenders in the patient a sense of support and partnership that is consistent with both the osteopathic and patient-centred models of healthcare. Finally, touch also has an ability to communicate professional virtues which patients search for in healthcare professionals including care, trust, boundaries and perhaps most importantly reassurance.

To conclude this project, chapter seven will now review some of the strengths and limitations of the current research project. This will be followed by a discussion of directions for future research and an exploration of the implications that the current research has for osteopathic practice and education. 


\section{Chapter Seven: Concluding Thoughts}


Chapter seven will begin by examining the academic boundaries of the study by outlining its strengths and limitations. This is followed by an exploration of the questions that have arisen from the study and directions for future research. The chapter will conclude with a brief discussion of the project's implications for osteopathic education and practice.

\section{Strengths of the Study}

The current study provides a rich and comprehensive exploration of the experience of osteopathic touch from the patient's perspective. It also offers osteopaths and osteopathic educators some research with which to support their own experience and anecdotal evidence.

One strength of this study is that it investigates a phenomenon that has had very little attention in osteopathic research. As mentioned earlier, while osteopathic literature emphasises the importance of touch to both the practitioner and the patient, osteopathic authors have predominantly utilised research from other disciplines to support their anecdotal experiences (Lederman, 2005; Milne, 1995; Nathan, 1999). While this approach is informative and enlightening it fails in some respects to attend to the subtle and complex nature of touch within an osteopathic session.

Given the scarcity of research into touch and the nature of the research aims (i.e. investigating human experience) a phenomenological method was employed to complete the study. As already discussed phenomenological research sets out to examine the nature of the lived experience of a phenomenon. Thus it is suggested that another strength of this study is that there is a consistency between the goals of the research and the philosophical and methodological approach utilised to complete the project. As has been discussed, phenomenological authors suggest that this consistency increases the rigour and reliability of the research. 
A further strength of this study is that due to the multi-disciplined nature of touch the findings are potentially useful to a wide range of healthcare professionals. Most obviously perhaps the research may be useful to other physical therapists that utilise touch in a similar way to osteopathic practitioners. Given the strong communicative functions of touch, this research may also be potentially useful to other healthcare professionals who use touch less than physical therapists but who still have to interact physically with their patient's bodies.

\section{Limitations of the Study}

The current study involved a process of analysing and synthesising data from five interviews to distil a number of themes that appeared to best represent the participants' lived experience of touch. Given the small sample size and the purposive sampling method utilised for this study the findings from this research cannot be generalised to other populations or to the greater population. However consistent with the phenomenological approach, the goal of this research was not to produce generalisable findings, but to achieve a rich and in-depth understanding of the experience of touch within an osteopathic session.

Another perceived limitation of the study is that the number of participants used was not enough to achieve the phenomenological goal of an in-depth exploration of the phenomenon. As this research was completed in partial fulfilment of the Masters of Osteopathy I was limited by both the required magnitude of the study and the time required to complete the project. Furthermore, some phenomenological authors have suggested that the phenomenological researcher's goal should be to continue to collect data until no new data emerges (Morse \& Field, 1996). It is acknowledged that while there are consistencies across the interviews in this study, the complexity and breadth of this research topic make it difficult to determine whether this goal was actually achieved. 
This research set out to examine the experience of touch in an osteopathic session and can only offer a theory as to why the participants experienced the practitioner's touch in this way. One theory is that touch engenders a sense of social support to the patient. In an interesting study conducted by Edens, Lakin and Abel (1992), the authors found that neither the presence of a stranger nor physical touch attenuated cardiovascular reactions to stress. However the presence of social support was an important mediating variable in the participant's reaction to stress. What this research may suggest is that the practitioner is able to show social support through the medium of touch.

Given the time constraints on the completion of this study a further limitation of this study was the necessary exclusion of certain methodological processes that have been recommended to further ensure methodological rigour. For example, Sanders (2003) has suggested that the transcripts should be returned to the participants following the interview in order for them to validate the interview and confirm that the data reflects their view of the phenomena. In addition Colaizzi's sixth step requires researchers to return to the participants after data analysis to elicit their views on the essential structure of the phenomenon (cited in Sanders, 2003).

Touch is a complex and intrinsic element of the interaction and communication that occurs between an osteopath and their patient. While the narrow structure of this study was a necessary compromise for an in-depth examination of touch, it is not possible to draw any comprehensive conclusions about the interaction between touch and other non-verbal and verbal forms of communication within the osteopath-patient relationship.

It could also be argued that a further limitation of this study was the narrow demographic group used for the research. Given that touch is culturally mediated, it is not possible to draw conclusions about the implications for this research to any other group except New Zealanders of European descent. However, this was considered a necessary compromise when considering the scale of the study and the accessibility to this population. 


\section{Directions for Future Research}

The aim of this study was to bring to light some of the salient features of the experience of touch within an osteopathic session. As with all research however, it is fair to say that this project has asked more questions of the world than it has answered. To start with, there is the question of generalisability - do the experiences of the participants in this study accurately reflect the experiences of the wider population of osteopathic patients? While patient experience is impossible to capture or examine quantitatively, a need exists to continue to examine the types and meaning of touch to patients of osteopathic care.

Another area of investigation that requires further investigation is the practitioner's experience of touch. Attempting to understand this facet of osteopathic practice would then enable researchers and practitioners to understand and appreciate the subtle differences that exist in the perceived quality of touch that occurs in the osteopathic session.

In order to gain some useful insight into the experience of touch, the selection criteria of this study excluded individuals who did not identify with being of New Zealand European descent. As detailed earlier, touch is culturally mediated and large differences exist in the way in which people of different cultures experience physical contact (Ayonrinde, 2003). Given the multicultural society that New Zealanders live, it would be useful for New Zealand osteopaths to have access to research that examined the experience of touch for other cultures living in New Zealand.

Due to the exploratory nature of this project the current study did not examine or investigate any potential gender differences in the experience of touch. Research in the psychological literature suggests that touching behaviour varies with the gender of both the toucher and those being touched (DiBiase \& Gunnoe, 2004; Remland et al., 1995). According to Remland et al. (1995):

Observed differences between males and females typically are attributed to the more affiliative $[$ sic] and/or more submissive sex roles of women in society 
compared to men; presumably, such roles lead women to establish closer proximity to others, to use a more direct body orientation and to be more receptive to the use of touch than men are (Remland et al., 1995, p. 282).

While these findings largely relate to public displays of touching behaviour, it does suggest some fundamental differences in the way in which men and women experience touch. Thus, it would be useful for future research to compare the experiences of touch between males and females in an osteopathic session.

Finally, the current study highlighted the important role played by touch in the communicative process. Moreover this research supported the literature that already exists on the patient-practitioner interaction. It would be useful for future research to examine other forms of non-verbal communication in the patient-practitioner interaction and explore how these shape and maintain a healthy therapeutic relationship in an osteopathic session.

\section{Implications for Education and Practice}

As discussed earlier, the qualitative nature of this research means that it is impossible to make any broad generalisations from the findings of the current study. However, as well as highlighting areas for future research the current study has drawn attention to the significance of touch for both healthcare education and healthcare practice.

This study has contributed to the growing body of evidence that illustrates the integral role touch plays in the patient-practitioner interaction. Moreover, this research suggests that from the moment the practitioner physically engages with the patient's body a contract is drawn which mediates and guides the therapeutic interaction. Significantly, research has shown that the perceived success of this interaction is greatly determined by the strength of the patient-practitioner alliance (D. Williams, 1997), which is built on and supported by many of the qualities that the current research has suggested are conveyed by touch. 
For teachers and educators within osteopathy and other physical therapies, this research has drawn attention to the importance of teaching effective and sensitive communication skills to students. Many of the qualities that the participants appreciated in their practitioners including engaging, listening, caring, supporting and trusting, are engendered in the participants through an open and competent interpersonal communication style. Thus, it is imperative that students are taught clear and effective communication skills. Moreover, the importance of this goal is evidenced by the universality of this finding in healthcare literature (Gyllensten et al., 1999; Jensen et al., 2000; Kelly, 2007; Roger et al., 2002).

This research also suggests that more emphasis needs to be placed on the role of touch within osteopathic and manual therapy training. Teachers and educators may need to think about educating students on the different types of touch, the communicative power of touch, the meaning of touch and the experience of touch for patients. This implication is consistent with a number of other authors who have similarly drawn attention to the importance of educating students about the role of touch within healthcare practice (Helm et al., 1997; Nathan, 2000; Posthuma, 1985; Roger et al., 2002). Furthermore, Helm et al. (1997) have recommended that the use of touch as a form of non-verbal communication should be discussed in continuing education courses and that course work should explore the importance of comforting, caring touch.

This research may also have implications for osteopathic and other physical therapy practice. Firstly, the current research project advocates the requirement for practitioners to be aware of how much information is conveyed, both consciously and subconsciously to their patients through the way in which they touch their bodies. The current research also suggests that understanding the types of touch that are possible within a therapeutic encounter could enhance a practitioner's use of both procedural and non-procedural affectional touch. Furthermore this research draws attention to the importance of non-verbal communication and suggests that practitioners need to reflect on and assess their own communication styles. This recommendation is also consistent with other research that suggests that practitioners should reflect on their communication skills and touching behaviours (Gyllensten et al., 1999; Potter et al., 2003; Roberts \& Bucksey, 2007). 
Finally, this research draws attention to the substantial amount of information that is communicated through touch. Given that conscious and sub-conscious motives and behaviours may be communicated to the patient through non-verbal behaviours such as touch, the current research suggests that osteopaths should examine and reflect on feelings and motivations they may bring into the treatment room. In order for this to be achieved, practitioners may need to use a clinical supervisor who can offer supervision around technical, emotional, interpersonal and intra-personal aspects of their practice. Moreover, it is suggested through this process, practitioners maybe able to be more present, honest, focused and open to their patients when they touch them and in this way provide them with a higher level of healthcare.

\section{A Final Word}

The current research is a hermeneutic enquiry into the lived experience of touch in an osteopathic session. An analysis of the participants' experience revealed that touch is an important feature of the practitioner-patient interaction. For the participants touch communicated the practitioner's care and attention as well as their professional attitude and competence. Examining the results of this research within the context of the developmental and healthcare literature revealed that touch is a powerful and distinctive form of communication. In addition, the healthcare literature indicates that effective communication forms the basis of a successful patient-practitioner relationship and profoundly dictates patient satisfaction and other healthcare outcomes.

A question that naturally follows from this conclusion however is why is touch such an important form of communication within healthcare? From this research it is apparent that touch communicates a number of virtues which participants identify as important attributes of healthcare professionals including truthfulness, trustworthiness, insight, respect, vulnerability, compassion and enthusiasm. Moreover, it appears that for the participants these intrinsic qualities of touch as well as its unmediated nature facilitates a natural and open dialogue between the practitioner's knowing hands and the patient's expressive body. 
References 
Ayonrinde, O. (2003). Importance of cultural sensitivity in therapeutic transactions: Considerations for healthcare providers [Electronic Version]. Disease Management and Health Outcomes, 11(4), 233-248.

Barnard, K. E., \& Brazelton, B. T. (Eds.). (1990). Touch: The foundation of experience. Madison, WI: International Universities Press.

Barnes, A. (2004). Am I a carer and do I care? An exploration of the concept of care as applied to osteopathic practice [Electronic Version]. Medicine, Health Care, and Philosophy, 7(2), 153-161.

Baron, R. J. (1992). Why aren't more doctors phenomenologists. In D. Leder (Ed.), The body in medical thought and practice (pp. 37-47). Dordrecht: Kluwer Academic Publishers.

Barrett, B., Muller, D., Rakel, D., Rabago, D., Marchand, L., \& Scheder, J. (2006). Placebo, Meaning, and health. Perspectives in Biology and Medicine, 49(2), 178-198. Retrieved February 29, 2008, from Health and Wellness Resource Centre database.

Barrier, P. A., Li, J. T. C., \& Jensen, N. M. (2003). Two words to improve physicianpatient communication: What else? Mayo Clinic Proceedings, 78(2), 211-214. Retrieved February 19, 2008, from Academic Search Premier database.

Barritt, P. (2005). Humanity in healthcare. Abingdon: Radcliffe Publishiing Ltd.

Bassett, C. (2002). Nurses' perceptions of care and caring. International journal of nursing practice, 8(1), 8-15. Retrieved February 9, 2007, from Academic Search Premier database.

Bendapudi, N. M., Berry, L. L., Frey, K. A., Parish, J. T., \& Rayburn, W. L. (2006). Patients' perspectives on ideal physician behaviors. Mayo Clinic Proceedings, 81(3), 338-344. Retrieved February 19, 2008, from Academic Search Premier database.

Billings, J. A., \& Stoeckle, J. D. (1999). The clinical encounter: A guide to the medical interview and case presentation (2nd ed.). Missouri: Mosby.

Blackwell, P. L. (2000). The influence of touch on child development: Implications for intervention. Infants and Young Children, 13(1), 28-39. Retrieved February 9, 2007 from Academic Search Premier database.

Bowers, L. J. (2000). Intimate strangers: Issues of touch. Topics in Clinical Chiropractic, 7(3), 11-18. Retrieved February 4, 2007, from Academic Search Premier database.

Bowlby, J. (1969). Attachment and loss (Vol. 1 - Attachment). London: The Hogarth Press and the Institute of Psycho-Analysis. 
Bowlby, J. (1988). A secure base - clinical applications of attachment theory. London: Tavistock/Routledge.

Cardy, I. (2004). Experience in stillness: A hermeneutic study of the breath of life in the cranial field of osteopathy. Unpublished Masters thesis, Unitec, Auckland, New Zealand.

Chang, S. O. (2001). The conceptual structure of physical touch in caring. Journal of Advanced Nursing, 33(6), 820-827. Retrieved February 10, 2008, from Academic Search Premier database.

Cohen, M. Z. (2006). Introduction to Qualitative Research. In G. LoBiondo-Wood \& J. Haber (Eds.), Nursing research: Methods and critical appraisal for evidencebased practice (6th ed., pp. 131-147). St Louis: Mosby Elsevier.

Comeaux, Z. (2005). Zen awareness in the teaching of palpation: An osteopathic perspective. Journal of Bodywork and Movement Therapies, 9(4), 318-326.

Conde-Agudelo, A., Diaz-Rossello, J. L., \& Belizan, J. M. (2003). Kangaroo mother care to reduce morbidity and mortality in low birthweight infants. Cochrane Database of Systematic Reviews, (2). Retrieved February 7, 2007, from http://www.mrw.interscience.wiley.com/cochrane/clsysrev/articles/CD002771/fr ame.html

Consedine, M. (2007). The Mask. Unpublished Manuscript.

Cooper, C. (1996). The art of reassurance. Australian Family Physician, 25(5), 697-698.

Crellin, J. K. (2005). Public expectations and Physician's responsibilities - Voices of medical humanities. Abingdon: Radcliffe Publishing Ltd.

Denzin, N. K., \& Lincoln, Y. S. (1998). Introduction - Entering the field of qualitative research. In N. K. Denzin \& Y. Lincoln (Eds.), Strategies of qualitative enquiry (pp. 1-34). Thousand Oaks: Sage Publications.

DiBiase, R., \& Gunnoe, J. (2004). Gender and culture differences in touching behavior. Journal of Social Psychology, 144(1), 49-62. Retrieved February 18, 2008, from Academic Search Premier database.

DiMenna, L. (2006). Considerations for implementation of a neonatal kangaroo care protocol. Neonatal Network, 25(6), 405-412. Retrieved February 28, 2007, from Academic Search Premier database.

Donalek, J. G., \& Soldwisch, S. (2004). Demystifying nursing research: An introduction to qualitative research methods. Urologic Nursing, 24(4), 354-356. Retrieved February 15, 2008, from MEDLINE database.

Donnelly, G. F. (2004). The placebo effect and holistic interventions. Holistic Nursing Practice, 18(5), 238-241. 
Dorland, W. A. N. (2003). Dorland's Illustrated Medical Dictionary (30th ed.). Philadelphia: W. B. Saunders.

Edens, J. L., Larkin, K. T., \& Abel, J. L. (1992). The effect of social support and physical touch on cardiovascular reactions to mental stress. Journal of Psychosomatic Research, 36(4), 371-381. Retrieved March 9, 2008, from ScienceDirect College Edition database.

Edwards, S. C. (1998). An anthropological interpretation of nurses' and patients' perceptions of the use of space and touch. Journal of Advanced Nursing, 28(4), 809-817. Retrieved February 9, 2007, from Academic Search Premier database.

Ekerholt, K., \& Bergland, A. (2004). The first encounter with Norwegian psychomotor physiotherapy: Patients' experiences, a basis for knowledge. Scandinavian Journal of Public Health, 32(6), 403-410. Retrieved February 2, 2007, from Academic Search Premier database.

Ekerholt, K., \& Bergland, A. (2006). Massage as interaction and a source of information. Advances in Physiotherapy, 8(3), 137-144. Retrieved February 2, 2007, from Academic Search Premier database.

Estabrooks, C. A. (1989). Touch: A nursing strategy in the intensive care unit. Heart and Lung: Journal of Critical Care, 18(4), 392-401. Retrieved February 25, 2007, from ScienceDirect College Edition database.

Estabrooks, C. A., \& Morse, J. M. (1992). Toward a theory of touch: the touching process and acquiring a touching style. Journal of Advanced Nursing, 17(4), 448-456. Retrieved February 25, 2007, from Academic Search Premier database.

Fagerberg, C. R., Kragstrup, J., Støvring, H., \& Rasmussen, N. K. (1999). How well do patient and general practitioner agree about the content of consultations? Scandinavian Journal of Primary Health Care, 17(3), 149-152. Retrieved March 7, 2008, from Academic Search Premier database.

Fareed, A. (1996). The experience of reassurance: Patients' perspectives. Journal of Advanced Nursing, 23(2), 272-279. Retrieved February 9, 2007, from Academic Search Premier database.

Field, T. (1998). Touch therapy effects on development [Electonic Version]. International Journal of Behavioral Development, 22(4), 779-797.

Field, T. (2000). Touch therapy. Edinburgh: Churchill Livingstone.

Field, T. (2001). Touch. Cambridge: Massachusetts Institute of Technology Press.

Finfgeld-Connett, D. (2006). Meta-synthesis of presence in nursing. Journal of Advanced Nursing, 55(6), 708-714. Retrieved February 27, 2008, from Academic Search Premier database. 
Finfgeld-Connett, D. (2008). Meta-synthesis of caring in nursing. Journal of Clinical Nursing, 17(2), 196-204. Retrieved February 23, 2008, from Academic Search Premier database.

Frank, L. K. (1957). Tactile communication [Electronic Version]. Genetic Psychology Monographs, 56, 209-225.

Fredriksson, L. (1999). Modes of relating in a caring conversation: A research synthesis on presence, touch and listening. Journal of Advanced Nursing, 30(5), 11671176. Retrieved February 9, 2007, from Academic Search Premier database.

Friedman, H. S. (1979). Non-verbal communication between patients and medical practitioners. Journal of Social Issues, 35(1), 82-99. Retrieved February March 8, 2008, from Academic Search Premier database.

Fung, C. H., Elliott, M. N., Hays, R. D., Kahn, K. L., Kanouse, D. E., McGlynn, E. A., et al. (2005). Patients' preferences for technical versus interpersonal quality when selecting a primary care physician. Health Services Research, 40(4), 957977. Retrieved February 27, 2008, from MEDLINE database.

Gleeson, M., \& Timmins, F. (2004). The use of touch to enhance nursing care of older person in long-term mental health care facilities. Journal of Psychiatric and Mental Health Nursing, 11(5), 541-545. Retrieved February 9, 2007, from Academic Search Premier database.

Gleeson, M., \& Timmins, F. (2005). A review of the use and clinical effectiveness of touch as a nursing intervention [Electronic Version]. Clinical Effectiveness in Nursing, 9(1-2), 69-77.

Golberg, B. (1998). Connection: An exploration of spirituality in nursing care. Journal of Advanced Nursing, 27(4), 836-842. Retrieved February 9, 2007 from Academic Search Premier database.

Goodman, J. E., \& Monaghan, L. (2007). Introduction. In L. Monaghan \& J. E. Goodmand (Eds.), A cultural approach to interpersonal communication (pp. 15). Melbourne: Blackwell Publishing Ltd.

Greene, A. U. (2001). Conscious mind - conscious body. Journal of Analytical Psychology, 46, 565-590. Retrieved February 6, 2007 from Academic Search Premier database.

Greenfield, B. H. (2006). The meaning of caring in five experienced physical therapists. Physiotherapy Theory and Practice, 22(4), 175-187. Retrieved February 23, 2008, from Academic Search Premier database.

Gyllensten, A. L., Gard, G., Hansson, L., \& Ekdahl, C. (2000). Interaction between patient and physiotherapist in psychiatric care: The physiotherapist's perspective. Advances in Physiotherapy, 2(4), 157-167. Retrieved February 21, 2008, from Academic Search Premier database. 
Gyllensten, A. L., Gard, G., Salford, E., \& Ekdahl, C. (1999). Interaction between patient and physiotherapist: a qualitative study reflecting the physiotherapist's perspective. Physiotherapy Research International, 4(2), 89-109. Retrieved February 15, 2008, from Academic Search Premier database.

Haber, J. (2002). Developing research questions and hypotheses. In G. LoBiondo-Wood \& J. Haber (Eds.), Nursing research: Methods and critical appraisal for evidence based practice (6th ed.). Missouri: Mosby Elsevier.

Harlow, H. F. (1958). The Nature of Love. American Psychologist, 13, 573-685.

Hartman, L. (1997). Handbook of osteopathic technique (3rd ed.). London: Chapman \& Hall.

Helm, J. S., Kinfu, D., Kline, D., \& Zappile, M. (1997). Acquisition of a touching style and the clinician's use of touch in physical therapy. Journal of Physical Therapy Education, 11(1), 17-25. Retrieved February 15, 2007, from CINAHL Plus database.

Hertenstein, M. J. (2002). Touch: Its communicative functions in infancy. Human Development, 45, 70-94. Retrieved May 16, 2006, from Academic Search Premier database.

Hertenstein, M. J., Verkamp, J. M., Kerestes, A. M., \& Holmes, R. M. (2006). The communicative functions of touch in humans, nonhuman primates, and rats: A review and synthesis of the empirical research [Electronic Version]. Genetic, Social, and General Psychology Monographs, 132(1), 5-94.

Holmes, J. (1993). John Bowlby and attachment theory. London: Routledge.

Hupcey, J. E., \& Miller, J. (2006). Community dwelling adults' perception of interpersonal trust vs. trust in health care providers. Journal of Clinical Nursing, 15(9), 1132-1139.

Hupcey, J. E., Penrod, J., Morse, J. M., \& Mitcham, C. (2001). An exploration and advancement of the concept of trust. Journal of Advanced Nursing, 36(2), 282293. Retrieved February 25, 2008, from Academic Search Premier database.

Janesick, V., J. (1998). The dance of qualitative research design: method, methodolotry, and meaning. In N. R. Denzin \& Y. S. Lincoln (Eds.), Strategies of qualitative inquiry (pp. 35-55). Thousand Oaks: Sage Publications.

Janesick, V., J. (2000). The choreography of qualitative research design. In N. R. Denzin \& Y. S. Lincoln (Eds.), Handbook of qualitative research (2nd ed., pp. 379-401). Thousand Oaks: Sage Publications.

Jensen, G. M., Gwyer, J., Shepard, K. F., \& Hack, L. M. (2000). Expert practice in physical therapy. Physical Therapy, 80(1), 28-52. 
Johns, J. L. (1996). A concept analysis of trust. Journal of Advanced Nursing, 24(1), 7683. Retrieved February 25, 2008, from Academic Search Premier database.

Jones, S. E. (1994). The right touch: Understanding and using the language of physical contact. New Jersey: Hampton Press.

Kaptchuk, T. J. (2002). The placebo effect in alternative medicine: Can the performance of a healing ritual have clinical significance? [Electronic Version] Annals of Internal Medicine, 136(11), 817-825.

Keller, V. F., \& Carroll, J. G. (1994). A new model for physician - patient communication. Patient Education and Counseling, 23(2), 131-140. Retrieved February 19, 2008, from ScienceDirect College Edition database.

Kelly, M. E. (2007). A practical guide for teachers of communication skills: A summary of current approaches to teaching and assessing communication skills. Education for Primary Care, 18(1), 1-10. Retrieved February 19, 2008 from Academic Search Premier database.

Koch, T. (1994). Establishing rigour in qualitative research: the decision trail. Journal of Advanced Nursing, 19(5), 976-986. Retrieved May 20, 2006, from Academic Search Premier database.

Koch, T. (1995). Interpretative approaches in nursing research: The influence of Husserl and Heidegger. Journal of Advanced Nursing, 21, 827-836. Retrieved May 20, 2006, from Academic Search Premier database.

Koch, T. (1996). Implementation of a hermeneutic inquiry in nursing: Philosophy, rigour and representation. Journal of Advanced Nursing, 24, 174-184. Retrieved May 20, 2006 from Academic Search Premier database.

Kuchera, M. L. (2005). Osteopathic manipulative medicine considerations in patients with chronic pain. Journal of the American Osteopathic Association, 105(9 SUPPL. 4).

Kumlin, I. W., \& Kroksmark, T. (1992). The first encounter. Physiotherapists' conceptions of establishing therapeutic relationships. Scandinavian Journal of Caring Sciences, 6(1), 37-44. Retrieved January 15, 2008, from Academic Search Premier database.

Kyle, T. V. (1995). The concept of caring: a review of the literature. Journal of Advanced Nursing, 21(3), 506-514. Retrieved February 9, 2007 from Academic Search Premier database.

Laakso, V., \& Niemi, P. M. (2004). Why do patients get relieved or remain worried during a primary care consultation? Psychology and Health, 19(Suppl. 1), 100101. Retrieved February 29, 2008, from Academic Search Premier database. 
Laverty, S. M. (2003). Hermeneutic phenomenology and phenomenology: A comparison of historical and methodological considerations [Electronic Version]. International Journal of Qualitative Methods, 2. Retrieved 26 April 2006 from http://www.ualberta.ca/ iiqm/backissues/2_3final/pdf/laverty.pdf.

Leder, D. (1992). A tale of two bodies: The Cartesian corpse and lived body. In D. Welton (Ed.), Body and flesh (pp. 117-129). Malden: Blackwell Publishing.

Lederman, E. (2005). The science and practice of manual therapy (2nd ed.). London: Elsevier.

Lee-Treweek, G. (2002). Trust in complementary medicine: The case of cranial osteopathy [Electronic Version]. Sociological Review, 50(1), 48-68.

Leijssen, M. (2006). Validation of the body in psychotherapy. Journal of Humanistic Psychology, 46(2), 126-146. Retrieved May 20, 2006, from Sage Premier database.

Liamputtong, P., \& Ezzy, D. (2005). Qualitative research methods (2nd ed.). Melbourne: Oxford University Press.

Liehr, P. R., \& LoBiondo-Wood, G. (2006). Qualitative approaches to research. In G. LoBiondo-Wood \& J. Haber (Eds.), Nursing research: Methods and critical appraisal for evidence-based practice (6th ed., pp. 148-175). Missouri: Mosby: Elsevier.

Lincoln, Y., \& Guba, E. G. (2000). Pragmatic controversies, contradictions and emerging confluences. In N. R. Denzin \& Y. S. Lincoln (Eds.), Handbook of qualitative research (2nd ed., pp. 163-189). Thousand Oaks: Sage Publications.

Lucas, N. (2005). Psychosocial factors in osteopathic practice: To what extent should they be assessed? International Journal of Osteopathic Medicine, 8(2), 49-59.

Makely, S. (2005). Professionalism in health care: A primer for career success. New Jersey: Pearson Prentice Hall.

May, S. J. (2001). Patient satisfaction with management of back pain. Part 1: What is satisfaction? Review of satisfaction with medical management. Physiotherapy, 87(1), 4-5.

McCain, G. C., Ludington-Hoe, S. M., Swinth, J. Y., \& Hadeed, A. J. (2005). Heart rate variability responses of a preterm infant to Kangaroo care [Electronic Version]. Journal of Obstetric, Gynecologic, and Neonatal Nursing, 34(6), 689-694.

McCann, K., \& McKenna, H. P. (1993). An examination of touch between nurses and elderly patients in a continuing care setting in Northern Ireland. Journal of Advanced Nursing, 18(5), 838-846. Retrieved February 9, 2007 from Academic Search Premier database. 
McDaniel, E., \& Andersen, P. A. (1998). International patterns of interpersonal tactile communication: A field study. Journal of Nonverbal Behavior, 22(1), 59-73.

Mead, N., \& Bower, P. (2000). Patient-centredness: A conceptual framework and review of the empirical literature. Social Science and Medicine, 51(7), 10871110. Retrieved February 20, 2008, from ScienceDirect College Edition database.

Mead, N., \& Bower, P. (2002). Patient-centred consultations and outcomes in primary care: A review of the literature. Patient Education and Counseling, 48(1), 51-61. Retrieved February 20, 2008, from ScienceDirect College Edition database.

Mead, N., Bower, P., \& Hann, M. (2002). The impact of general practitioners' patientcentredness on patients' post-consultation satisfaction and enablement. Social Science and Medicine, 55(2), 283-299. Retrieved February 20, 2008, from ScienceDirect College Edition database.

Mechanic, D. (1998). The functions and limitations of trust in the provision of medical care. Journal of Health Politics, Policy and Law, 23(4), 661-686.

Merleau-Ponty, M. (1962). Phenomenology of perception (C. Smith, Trans.). New York: Humanities Press. Retrieved February 24, 2008, from Academic Search Premier database.

Milne, H. (1995). The heart of listening. California: North Atlantic Books.

Mitchell, N. (2005). The healing story : the narrative in osteopaths' interaction with their patients' bodypsyches. Unpublished Masters Thesis, Unitec, Auckland, New Zealand.

Montagu, A. (1986). Touching: The human significance of the skin (3rd ed.). New York: Harper \& Row.

Morse, J. M., \& Field, P. A. (1996). Nursing research: The application of qualitative approaches. London: Chapman \& Hall.

Nathan, B. (1993). Touching patients (or people). Journal of Osteopathic Education, $3(1), 29-31$.

Nathan, B. (1999). Touch and Emotion in Manual Therapy. Edinburgh: Churchill Livingstone.

Nathan, B. (2000). Concepts for osteopathic health care: Section 12: Touch. Unpublished Manuscript, British School of Osteopathy, London, United Kingdom. 
Nussbaum, G. B. (2003). Spirituality in critical care: Patient comfort and satisfaction. Critical Care Nursing Quarterly, 26(3), 214-220. Retrieved February 9, 2007, from Academic Search Premier database.

Ong, L. M. L., De Haes, J. C. J. M., Hoos, A. M., \& Lammes, F. B. (1995). Doctorpatient communication: A review of the literature. Social Science and Medicine, 40(7), 903-918. Retrieved February 29, 2008, from ScienceDirect College Edition database.

Osteopahic Council of New Zealand. (2006). The Osteopathic Council: Te Kaunihera Raweke Whenuaua O Aoteraoa: Code of Ethics. Wellington: Osteopathic Council of New Zealand.

Parsons, J., \& Marcer, N. (2006). Osteopathy: Models for diagnosis, treatment and practice. Edinburgh: Elsevier Limited.

Piasecki, M. (2003). Clinical communication handbook. Massachusetts: Blackwell Publishing.

Polit, D. F., \& Beck, C. T. (2004). Nursing research: Principles and methods (7th ed.). Philadelphia: Lippincott Williams \& Wilkins.

Polit, D. F., \& Hungler, B. P. (1999). Nursing Research: Principles and methods (6 $^{\text {th }}$ ed.). Philadelphia: Lippincott Williams and Wilkins.

Porter, R. (1997). The greatest benefit to mankind: A medical history of humanity from antiquity to the present. London: Harper Collins Publishers.

Posthuma, B. (1985). Learning to touch [Electronic Version]. Canadian Journal of Occupational Therapy 52(4), 189-193.

Potter, M., Gordon, S., \& Hamer, P. (2003). The physiotherapy experience in private practice: The patients' perspective [Electronic Version]. Australian Journal of Physiotherapy, 49(3), 195-202.

Priest, S. (1998). Merleau-Ponty: The arguments of the philosophers [electronic resource]. London: Routledge.

Purtilo, R., \& Haddad, A. (2007). Health professional and patient interaction (7th ed.). Missouri: Saunders Elsevier.

Pye, J., \& Jago, W. (1998). Effective communication in practice: A handbook for bodywork therapists. Edinburgh: Churchill Livingstone.

Ray, M. A. (1994). The richness of phenomenology: Philosophic, theoretic, and methodologic concerns. In J. M. Morse (Ed.), Critical issues in qualitative research methods (pp. 117-135). London: Sage Publications. 
Reifsteck, S. W. (1998). Difficult physician-patient relationships. Medical Group Management Journal, 45(5), 47-54. Retrieved February 19, 2008, from MEDLINE database.

Remland, M. S., Jones, T. S., \& Brinkman, H. (1995). Interpersonal distance, body orientation, and touch: Effects of culture, gender, and age. The Journal of Social Psychology, 135(3), 281-297. Retrieved February 18, 2008, from Academic Search Premier database.

Rethans, J. J., Sturmans, F., Drop, R., Van Der Vleuten, C., \& Hobus, P. (1991). Does competence of general practitioners predict their performance? Comparison between examination setting and actual practice [Electronic Version]. British Medical Journal, 303(6814), 1377-1380.

Rice, P. L., \& Ezzy, D. (1999). Qualitative Research Methods: A Health Focus. Melbourne: Oxford University Press.

Roberts, L., \& Bucksey, S. J. (2007). Communicating with patients: What happens in practice? Physical Therapy, 87(5), 586-594.

Roger, J., Darfour, D., Dham, A., Hickman, O., Shaubach, L., \& Katherine, S. (2002). Physiotherapist's use of touch in inpatient settings. Physiotherapy Research International, 7(3), 170-186. Retrieved February 7, 2007, from Academic Search Premier database.

Rojas, M. A., Kaplan, M., Quevedo, M., Sherwonit, E., Foster, L. B., Ehrenkranz, R. A., et al. (2003). Somatic growth of preterm infants during skin-to-skin care versus traditional holding: A randomized, controlled trial. Journal of Developmental and Behavioral Pediatrics, 24(3), 163-168.

Routasalo, P. (1996). Non-necessary touch in the nursing care of elderly people. Journal of Advanced Nursing, 23(5), 904-911. Retrieved February 10, 2007, from Academic Search Premier database.

Routasalo, P. (1999). Physical touch in nursing studies: A literature review. Journal of Advanced Nursing, 30(4), 843-850. Retrieved February 9, 2007, from Academic Search Premier database.

Russell, B. (1977). ABC of relativity. London: Allen and Unwin.

Sachs, F. (1988). The intimate sense. The Sciences, 28(1), 28-34.

Sanders, C. (2003). Application of Colaizzi's method: Interpretation of an auditable decision trail. Contemporary Nurse, 14(3), 292-302. Retrieved May 15, 2006 from EBSCO database.

Schneider, Z., Elliott, D., LoBiondo-Wood, G., \& Haber, J. (2003). Nursing research methods critical appraisal and utilisation (2nd ed.). Sydney: Mosby. 
Seffinger, M., King, H., Ward, R., Jones, J., Rogers, F., \& Patterson, M. (2003). Osteopathic Philosophy. In R. Ward (Ed.), Foundations for Osteopathic Medicine (pp. 3-18). Philadelphia: Lippincott Williams \& Wilkins.

Seidel, H. M., Ball, J. W., Dains, J. E., \& William Benedict, G. (2003). Mosby's guide to physical examination (5th ed.). St Louis: Mosby.

Silverman, J., Kurtz, S., \& Draper, J. (2005). Skills for communicating with patients. Oxford: Radcliffe Publishing Ltd.

Sim, J. (1986). Truthfulness in the therapeutic relationship. Physiotherapy Practice, 2(3), 121-127. Retrieved February 24, 2008, from Academic Search Premier database.

Soanes, C., \& Stevenson, A. (Eds.). (2006). Concise Oxford English Dictionary (11th, revised ed.). Oxford: Oxford University Press.

Sontag, S. (1977). Illness as metaphor. New York: Farrar, Straus and Giroux.

Stewart, M. A. (1995). Effective physician-patient communication and health outcomes: A review. Canadian Medical Association Journal, 152(9), 1423-1433. Retrieved February 20, 2008, from Academic Search Premier.

Stone, C. (1999). Science in the art of osteopathy: osteopathic principles and practice. Cheltenham: Nelson Thornes.

Streubert, H. J., \& Carpenter, D. R. (1999). Qualitative research in nursing: Advancing the humanistic imperative (2nd ed.). Philadelphia: Lippincott.

Sundin, K., \& Jansson, L. (2003). 'Understanding and being understood' as a creative caring phenomenon: In care of patients with stroke and aphasia. Journal of Clinical Nursing, 12(1), 107-116.

Talvitie, U. (2000). Socio-affective characteristics and properties of extrinsic feedback in physiotherapy. Physiotherapy Research International, 5(3), 173-188. Retrieved May 7, 2006, from Academic Search Premier database.

Thom, D. H. (2001). Physician behaviors that predict patient trust. Journal of Family Practice, 50(4), 323-328. Retrieved February 24, 2008, from Academic Search Premier database.

Thom, D. H., \& Campbell, B. (1997). Patient-physician trust: An exploratory study. Journal of Family Practice, 44(2), 169-176. Retrieved February 24, 2008, from Health and Wellness Resource Centre database.

Thomas, K. B. (1987). General practice consultations: Is there any point in being positive? [Electronic Version] British Medical Journal, 294(6581), 1200-1202. 
Tobin, G. A., \& Begley, C. M. (2004). Methodological rigour within a qualitative framework. Journal of Advanced Nursing, 48(4), 388-396. Retrieved March 5, 2008, from Academic Search Premier database.

Toronto, E. L. K. (2002). A clinician's response to physical touch in the psychoanalytic setting. International Journal of Psychotherapy, 7(1), 69-81. Retrieved May 7, 2006, from Academic Search Premier database.

Trojan, L., \& Yonge, O. (1993). Developing trusting, caring relationships: home care nurses and elderly clients. Journal of Advanced Nursing, 18(12), 1903-1910. Retrieved February 25, 2008, from Academic Search Premier database.

Tuckett, A. G. (2005). Part II. Rigour in qualitative research: complexities and solutions. Nurse Researcher, 13(1), 29-42. Retrieved from March 5, 2008, from Academic Search Premier database.

Van Dongen, E., \& Elema, R. (2001). The art of touching: the culture of 'body work' in nursing. Anthropolgy \& Medicine, 8(2/3), 149-162. Retrieved May 6, 2006 from Academic Search Premier database.

van Manen, M. (1997). Researching lived experience (2nd ed.). Ontario: The Althouse Press.

Ventegodt, S., Morad, M., \& Merrick, J. (2004). Clinical holistic medicine: Classic art of healing of the therapeutic touch. The Scientific World Journal, 4, 134-147.

Vickers, A., Ohlsson, A., Lacy, J. B., \& Horsley, A. (2004). Massage for promoting growth and development of preterm and/or low birth-weight infants. Cochrane Database of Systematic Reviews, (2). Retrieved February 5, 2007, from http://mrw.interscience.wiley.com/cochrane/clsysrev/articles/CD000390/frame.h $\underline{\mathrm{tml}}$

Walters, A. J. (1995). The phenomenological movement: implications for nursing research. Journal of Advanced Nursing, 22, 791-799. Retrieved June 5, 2006, from Academic Search Premier database.

Wilkin, K., \& Slevin, E. (2004). The meaning of caring to nurses: An investigation into the nature of caring work in an intensive care unit. Journal of Clinical Nursing, 13(1), 50-59. Retrieved February 9, 2007, from Academic Search Premier database.

Williams, D. (1997). Communication skills in practice - A practical guide for health professionals. London: Jessica Kingsley Publishers.

Williams, N. H. (2007). Optimising the psychological benefits of osteopathy. International Journal of Osteopathic Medicine, 10(2-3), 36-41.

Willis, J. W. (2007). Foundations for qualitative research: Interpretive and critical approaches. London: Sage Publications. 
References

Wyschogrod, E. (1981). Empathy and sympathy as tactile encounter. Journal of Medicine and Philosophy, 6(1), 25-43. 
Appendices 


\section{Appendix A - Ethical Approval}

\section{UUnitec}

pricree +6498494180 tax +6498152901 web www unitec.ac.nz acidess Caurington Rd, ML Albert. Private Bag 92025, Auckland. New Zealand

Seth Consedine

65 Wanganui Ave

Herne Bay

Auckland 1011

21 September 2006

Dear Seth

Your file number for this application: 2006.600

Title: Toward a Greater Understanding of the Physical Contact which occurs between an Osteopath and their Patient

Your application for ethics approval has been reviewed by the Unitec Research Ethics Committee (UREC) and has been approved for the following period:

Start date: 20 September 2006

Finish date: 30 September 2007

Please note that:

1. the above dates must be referred to on the information AND consent forms given to all participants

2. you must inform UREC, in advance, of any ethically-relevant deviation in the project. This may require additional approval.

This letter has been copied to the Principal Supervisor for Unitec student research projects.

You may now commence your research according to the protocols approved by UREC. We wish you every success with your project.

Yours sincerely

Fortix Rishnond

G. Dr Andrew Stewart

Deputy Chair, UREC

RMO refH: 814

cc: Dr Elizabeth Niven

Cynthia Almeida 


\section{Appendix B - Inclusion/Exclusion Criteria}

- Participants must be over the age of 25 years. This age minimum has been selected to ensure a sample that has an adequate level of maturity, reflection and insight as to be able to most inform the research process.

- Participants must have an unspecific diagnosis (i.e. no identified pathology or musculoskeletal diagnosis). Clients of osteopathy may come along to maintain their current level of health, rather than having a specific musculoskeletal issue which requires treatment. This criterion has been included because it is believed that participants who are not suffering from an unspecific diagnosis are more likely to be able to identify various elements of osteopathic touch (rather than focusing on their presenting condition).

- Participants must be of New Zealander European descent. Responses to touch are culturally bound and thus to ensure some homogeneity to the sample European New Zealanders have been chosen.

- Participants must have a sound grasp of the English language (English must be a first language). Intrinsic to the Heideggerian world understanding, language is the universal medium in which comprehension occurs for an individual (Koch, 1995; Laverty, 2003) and thus it is important for participants to be able to express themselves clearly and succinctly in their own native language.

- Participants will have seen an Osteopath from one of the local Osteopathic clinics which have been selected

- Participants must have had an osteopathic session in the 48 hours leading up to the interview. As participants will be asked to explain and interpret their experiences within their osteopathic session, it is important that participants are able to recollect as clearly as possible the physical contact which occurred between themselves and the Osteopath.

- The Osteopathic treatment received will have involved a number of different Osteopathic approaches (at least three) as confirmed by the residing practitioner (for example, cranial, visceral, functional etc.) These techniques will be noted by the researcher for each participant and confirmed with the presiding osteopath. As the type of physical contact can vary between different Osteopathic approaches, it is important to recruit participants who have received a variety of techniques which cover a range of approaches.

- Participants will not be healthcare professionals. These individuals will be excluded as healthcare professionals have generally had some training on the impact of touch in a medical setting and are thus likely to have generated their own views about touch within clinical practice. This knowledge may potentially prejudice their data. 


\title{
Appendix C - Information Sheet for Participants Unitec
}

\author{
Information for Participants
}

\section{Toward a Greater Understanding of the Physical Contact which occurs between an Osteopath and their} Patient

\section{The study}

I am conducting this study in an attempt to investigate and qualify the subjective experience of touch within an osteopathic session.

As with many forms of physical therapy touch plays an important role in the therapeutic relationship that develops between an Osteopath and their clients. Surprisingly, while this statement has an intuitive reality there remains a scarcity of quality research to support this idea.

In order to contribute to osteopathic research and expand practitioner knowledge, I would like to explore your views, experiences and understanding of the types of touch which you experienced during your osteopathic session.

\section{How you are able to participate}

To achieve these goals I will be interviewing participants about their experiences of touch within an osteopathic session that they have had within the last forty-eight hours. A semi-structured interview will be used, allowing an open discussion which will be guided by both the interviewer and interviewee. The interview is expected to last approximately one hour and will be audio-taped for accuracy and later review. The interviews will be conducted at a pre-arranged place and time, which is mutually convenient for both the participant and the primary researcher.

You are free to withdraw from the interview at any time and your interview script would be subsequently excluded from the study. Following the completion of the interview, you have two weeks in which to withdraw your interview script from the research process, as following this the interview script will be analysed and synthesized with other interviews. To withdraw you simply need to contact the primary researcher by phone on the contact details below.

Following the completion of the interviews, the data will be transcribed and interpreted within current touch and osteopathic literature. To complete the research process an article will be written and submitted for publication in Osteopathic journals.

\section{Confidentiality}

You will not be individually identified in any reports, as all comments will be attributed to a pseudonym which can only be identified by the primary researcher (myself). All my notes, data and the consent forms will be kept securely for 5 years from 2006 .

\section{Consent}

Participants will be asked to complete a consent form before the interview commences.

\section{Contact}

Please feel free to contact the researcher or supervisor if you have any queries or would like to participate in the research:

Primary Researcher - Seth Consedine (09) 3764557 (This contact number has an answer phone and a message can be left which will be returned ASAP)

Supervisor - Elizabeth Niven, (09) 815-4321 ext 8320

This study has been approved by the Unitec Research Ethics Committee from (August 2006) to (September 2007). If you have any complaints or reservations about the ethical conduct of this research, you may contact the Committee through the UREC Secretariat (Ph: 098154321 ext.7254). Any issues you raise will be treated in confidence and investigated fully, and you will be informed of the outcome. 


\section{Appendix D - Consent Form

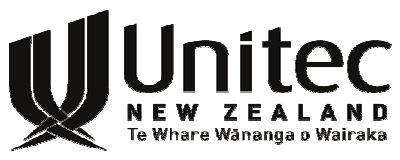

\section{Consent Form}

\section{TOWARD A GREATER UNDERSTANDING OF THE PHYSICAL CONTACT WHICH OCCURS BETWEEN AN OSTEOPATH AND THEIR PATIENT}

This consent form will help us obtain information for a research project examining the different components of touch which occur during an osteopathic session as experienced by the participant.

I have had the research project explained to me and I have read and understand the information sheet given to me.

I understand that taking part in this project is voluntary (my choice). I may withdraw from the project up until two weeks after the interview prior to the data analysis stage of the research.

I understand that everything I say is confidential and none of the information I give will identify me and that the only persons who will know what I have said will be the Primary Researcher Seth Consedine.

I also understand that all the information that I give will be stored securely on a computer at Unitec for a period of 5 years from 2006.

I understand that my discussion with the researcher will be taped and transcribed and no material could identify me if used in any future reports on this project.

I understand that I can see the finished research document.

I am aware that I may contact the Primary Researcher Seth Consedine on (09) 3764557 or the Co-ordinator/Supervisor of the research Elizabeth Niven at Unitec, (09) 815-4321 ext 8320 if I have any queries about the project.

I have had time to consider everything and I give my consent to be a part of this.

Participant Signature:

Date:

Project Researcher:

Date:

This study has been approved by the Unitec Research Ethics Committee from (August 2006 ) to (September 2007 ). If you have any complaints or reservations about the ethical conduct of this research, you may contact the Committee through the UREC Secretariat (Ph: 098154321 ext.7254). Any issues you raise will be treated in confidence and investigated fully, and you will be informed of the outcome. 


\section{Appendix E - Information about Researcher}
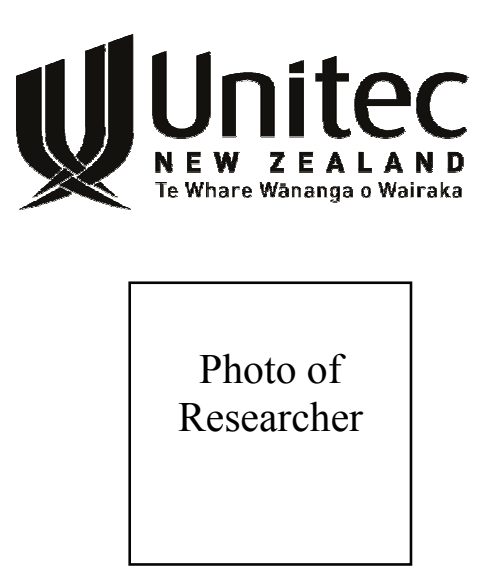

Hello,

My name is Seth Consedine and I am a 29year old Cantabrian who moved to Auckland four years ago to train as an Osteopath. I was drawn to osteopathy as a profession as I have a keen interest in people and believe osteopathy to be a form of healthcare which offers a unique, holistic perspective on the human condition.

At this stage of my training (final year of the Masters of Osteopathy) I am required to spend three half days working in the student clinic practising as a supervised Osteopath as well as write a research project on a subject that is relevant to the field of osteopathy. This research is a critical element in my training, which offers me a chance to explore an important area of osteopathy as well as develop my own understanding and appreciation of what healthcare and in particular osteopathy is about.

For my research I have decided to investigate the experience of touch within an osteopathic session. I believe that the significance of touch within osteopathy is often undervalued by both students and some practitioners. Furthermore, there is a shortage of well conducted research into touch and osteopathy.

The research would involve a forty five minute interview (approx.) with me which would focus on your experience of touch within an osteopathic session. As this is an interview about your experience, the information will of course be treated with the utmost respect. If you are interested in participating, please inform the receptionist/osteopath at the clinic of your phone number so that I may contact you to discuss the study.

Your participation in my research would be greatly appreciated.

Regards

Seth Consedine 


\section{Appendix F - Interview Schedule}

\section{Interview Schedule}

1. Welcome the participant. Thank them for their participation. Confirm to the participant what their involvement in this research will entail and approximately how long this will take. Ensure the participant understands both the information sheet and the consent form and reiterate their right to withdraw from the interview at any stage. The participant will then sign the consent form.

2. Explain to the participant that my research is attempting to investigate the element of human contact during an osteopathic session. Furthermore in order to achieve this I would like to discuss with them a recent osteopathic experience that they have had, in particular focusing on the physical contact which occurred between themselves and the osteopath.

3. The interview will have a semi-structured format, allowing an open and fluent discussion about human contact and the osteopathic session to develop between the participant and the interviewer. Throughout the interview, I will of course be alert to any discomfort or awkwardness that surfaces and respond appropriately, for example by checking their agreement to continue; reminding them of the confidential nature of the interview and research; moving to a safer topic; explaining the availability of counselling; and if necessary discontinuing the interview .

4. It is expected that the interview will cover elements of both procedural (handshaking, examination, palpation and osteopathic technique) and non-procedural (that used to express kindness, sympathy or caring) touch as well as the participants thoughts on the significance of this type of human contact. If particular areas of human contact and the osteopathic session are not discussed throughout the interview I would like to raise these ideas to the participant at the end of the interview.

Thus I would like to ensure that both procedural and non-procedural touch; touch used in other clinical settings; and any thoughts the participant would like to share with a training osteopath, are all discussed in the interview. 